University of South Carolina

Scholar Commons

1997

\title{
Molecular-level modeling of the viscoelasticity of crosslinked polymers: Effect of time and temperature
}

\author{
Philip P. Simon \\ University of South Carolina - Columbia \\ Harry J. Ploehn \\ University of South Carolina - Columbia, ploehn@cec.sc.edu
}

Follow this and additional works at: https://scholarcommons.sc.edu/eche_facpub

Part of the Chemical Engineering Commons

\section{Publication Info}

Journal of Rheology, 1997, pages 641-670. Copyright 1997 American Institute of Physics. This article may be downloaded for personal use only. Any other use requires prior permission of the author and the American Institute of Physics.

The following article appeared in (citation of published article) and may be found at http://dx.doi.org/ $10.1122 / 1.550828$.

DOI:10.1122/1.550828

This Article is brought to you by the Chemical Engineering, Department of at Scholar Commons. It has been accepted for inclusion in Faculty Publications by an authorized administrator of Scholar Commons. For more information, please contact digres@mailbox.sc.edu. 


\title{
Molecular-level modeling of the viscoelasticity of crosslinked polymers: Effect of time and temperature
}

\author{
Philip P. Simon and Harry J. Ploehn ${ }^{\text {a) }}$ \\ Department of Chemical Engineering, University of South Carolina, \\ Swearingen Engineering Center, Columbia, South Carolina 29208
}

(Received 18 September 1996; final revision received 18 February 1997)

\section{Synopsis}

We present a new molecular-level picture of chain dynamics for describing the viscoelasticity of crosslinked polymers. The associated mathematical model consists of a time-dependent momentum balance on a representative polymer segment in the crosslinked network, plus phenomenological expressions for forces acting on the segments. These include a cohesive force that accounts for intermolecular attraction, an entropic force describing the thermodynamics governing chain conformations, and a frictional force that captures the temperature dependence of relative chain motion. We treat the case of oscillatory uniaxial deformation. Solution of the model equations in the frequency domain yields the dynamic moduli as functions of temperature and frequency. The model reproduces all of the qualitative features of experimental dynamic modulus data across the complete spectrum of time and temperature, spanning the glassy zone, the $\beta$ transition, the dynamic glass transition, and the rubbery zone. All of the model parameters can be evaluated through the use of independent experimental data. Comparison of model predictions with experimental data yields good quantitative agreement outside of the glass transition region. (C) 1997 The Society of Rheology. [S0148-6055(97)01403-X]

\section{INTRODUCTION}

Crosslinked polymers, such as epoxy resins, serve in structures subjected to timedependent mechanical loading with potentially large variations in temperature and plasticizer concentration. Designing optimal materials for specific applications requires an understanding of the effects of time, temperature, and plasticizer on the viscoelastic moduli of crosslinked polymers. In this paper, we postulate a molecular-level mechanism for the viscoelasticity of fully crosslinked polymers and construct a model that rationalizes the effects of time and temperature on observed uniaxial elongation moduli spanning the glassy and rubbery regimes. The effect of plasticizer content will be treated in a subsequent publication.

Crosslinked polymers behave like linearly elastic materials under some conditions [Adams and Miller (1977); Pomies and Carlsson (1994); Tsotsis and Weitsman (1990)], but also display significant viscoelastic responses over large temperature ranges and time scales [Miyase et al. (1993a); Miyase et al. (1993b); Crossman et al. (1978); Brinson and Knauss (1991)]. At low temperatures and short times, crosslinked polymers exhibit a glassy response, which may include a $\beta$ or $\delta$ transition due to relaxations associated with local vibrational and rotational motion. At high temperatures and long time scales, they

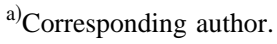


display rubbery behavior. The $\alpha$ transition, or glass transition, separates the glassy and rubbery regimes. Crosslinked polymers do not display a terminal zone at high temperatures and long time scales wherein the relaxation modulus decreases to zero.

A number of rigorous molecular models exist for uncrosslinked polymer solutions [Rouse (1953)] and polymer melts [Doi and Edwards (1986)]. These models postulate that applied deformations alter polymer chain conformations, thus, creating entropic forces manifested as stress in the material. Random thermal motions of the chains (e.g., reptation) may dissipate some of the strain energy and relax the forces over time. However, the reptation model [Doi and Edwards (1986)] cannot treat fully crosslinked polymers because it postulates relaxation by axial diffusion of chain ends out of their entanglement tubes. Adaptations of these models for crosslinked polymers [Tsenoglou (1986)] attribute the viscoelasticity to dangling chains resulting from incomplete crosslinking. Earlier, Kirkwood (1946) and Mooney (1959) developed Brownian relaxation models to describe the viscoelasticity of fully crosslinked polymers. None of the aforementioned models account for van der Waals forces, which dominate the glassy regime; thus, they can only describe the viscoelastic response around the rubbery regime. We are unaware of any molecular models that can describe the whole time-temperature spectrum of crosslinked polymer mechanical behavior.

Phenomenological spring and dashpot models comprise the only methodology currently available for rationalizing the viscoelastic moduli of crosslinked polymers spanning the glassy and rubbery regimes. Although these models successfully correlate data, they have a number of deficiencies. First, their parameters lack physical significance. Because the relaxation times are chosen arbitrarily, different parameter sets may produce good fits of a given experimental data set. More importantly, the equations only account for time dependence, thus, requiring data taken at constant temperature and plasticiser content.

Measurements of polymer dynamic properties typically employ either isothermal frequency sweeps (isotherms) or isochronal temperature sweeps (isochrones). Isotherms cannot capture all of the material's viscoelastic behavior in a single experiment because of practical limits on deformation frequency. Time-temperature superpositioning (TTS) has been used successfully to predict viscoelastic properties outside of the accessible range of time and temperature. However, the accuracy, as well as the validity of TTS, has been questioned [Plazek (1968)].

On the other hand, isochronal measurements are useful because they reveal all of the salient features of material viscoelastic behavior (glassy and rubbery regimes, various transitions) in a single experiment spanning an accessible range of temperatures. Furthermore, no subjective manipulation of the data is required. Unfortunately, there are no equations from either molecular or phenomenological models that can rationalize isochrones with moduli spanning two orders of magnitude over a modest range of temperatures.

The primary difficulty in modeling polymer behavior over large domains of time and temperature is in incorporating various forces and mechanisms within a consistent theoretical framework. van der Waals forces responsible for glassy behavior act over atomic length scales. Multiatom bond rotations must be described to account for $\beta$ relaxations. Macromolecular reconfiguration, described using bead-spring or reptation models, must be invoked to predict the rubbery modulus. The widely varying length scales of these phenomena represent a considerable challenge to efforts to formulate a comprehensive model.

The model presented in this paper attempts to include all of the aforementioned mechanisms within a molecular context. In this model, a crosslinked polymer chain is 
constrained at its ends by immobile crosslinks and along its contour by an entanglement tube formed by neighboring chains. Neighboring chains limit the lateral motion of the test chain. At certain locations, this constraint may be strong enough to eliminate all lateral motion, thus, permitting only axial chain motion. These locations, called entanglement junctions, divide the tube into subtubes and the chain into shorter strands. Although the chain is immobilized at the crosslinked end points, the constituent strands may redistribute their lengths among the subtubes through reptation in response to the forces acting upon them.

The mathematical formulation consists of a time-dependent momentum balance on a representative crosslinked chain subjected to cohesive, entropic, and frictional forces. By drawing on linear elasticity theory, the model incorporates the intermolecular cohesive force by introducing the concept of mechanical volume (a pressure-induced dilatation), which enables a description of both the magnitude of the cohesive force as well as the underlying mechanism for the $\beta$ relaxation. The model accounts for the temperature dependence of interchain frictional forces using the concept of thermal free volume (thermally induced dilatation). Ideally, a single equation of state (EOS) should provide both the mechanical and thermal-free volumes. Since we lack a suitable EOS spanning the glassy and rubbery regimes, we treat the mechanical and thermal-free volumes as independent quantities. Nevertheless, the need for these two types of free-volume points to the interrelationship between the polymer's thermodynamic state and its dynamic response, as well as to the fundamental distinction between the dilatational and viscoelastic glass transitions.

The model equations, solved in either the time or frequency domain, can be used to calculate isothermal frequency sweeps as well as isochronal temperature sweeps. For mathematical convenience, we use a transform technique to analyze exclusively the frequency domain response.

\section{GLASS TRANSITION MODEL FOR CROSSLINKED POLYMERS}

\section{A. Molecular view}

Several types of forces act on polymer chains in a glass, rubber, or melt. The hard-core Born repulsion prevents overlap of molecules. The van der Waals attractive force binds the chains into a cohesive bulk. An applied deformation alters the distribution of intermolecular separations and, thus, the magnitude and direction of the forces acting on each chain. The work done in applying the deformation represents potential energy stored as strain energy. The random thermal motions of a chain, in cooperation with the motions of neighboring chains, restore the equilibrium distribution of intermolecular separations and chain end-to-end distances, relax the forces exerted on chains, and dissipate stored strain energy.

The present model treats these various molecular phenomena in different ways. We use the entanglement tube concept [Doi and Edwards (1986)] to describe the repulsive forces that constrain the lateral motion of chains. An applied deformation distorts the entanglement tube, alters the distribution of separation distances between parts of the enclosed chain and its neighbors, and, thus, changes the mutual van der Waals attractive forces. The resultant of these forces, which we call the cohesive force, can be quantified using the concept of "mechanical volume."' Mechanical volume represents space within the entanglement tube in excess of the equilibrium space occupied by the chain in the undeformed state at constant temperature. The applied deformation also creates a nonequilibrium distribution of chain conformations. The entropic force is an effective thermodynamic force that biases the thermal motion of chains so as to restore the equilibrium 
distribution of conformations. The relative motion of neighboring chains also creates a frictional force that dissipates stored strain energy as heat.

We view the crosslinked polymer as a network of flexible chains, each crosslinked to the network at its ends. In the high-temperature limit, thermal energy dominates all intermolecular interactions, and so the chains are effectively unconstrained by neighbors. In this state, the phantom chain models in the classical theory of rubber elasticity [James and Guth (1943); Flory (1953)] describe the polymer behavior. At lower temperatures, chain interactions produce entanglements, leading to viscoelasticity and glassy behavior.

We postulate two kinds of entanglements: the entanglement tube and the entanglement junction [Fig. 1(a)]. Neighboring chains impose topological constraints that restrict the configurations available to a given chain. Recent rubber elasticity theories [e.g., Gaylord (1979)] represent such entanglements by restricting the volume available to a chain to that of an entanglement tube. The chain may assume all possible configurations within the confines of its entanglement tube. The tube is a mean-field representation of the confining effect of repulsive intermolecular interactions [Doi and Edwards (1986)].

The cross section of the entanglement tube varies along the length of the chain as a function of the interactions with the neighboring chains. Locations with stronger interactions between the chain and its neighbors correspond to smaller tube cross-sectional areas. Locations where the cross section of the tube is comparable to the cross section of the chain are called entanglement junctions. Entanglement junctions have several important characteristics. First, they act like crosslinks insofar as they prohibit chain motion in lateral directions. Junctions divide the chain into strands and the tube into subtubes. Entanglement junctions differ from crosslinks because they permit the chain to slide axially through them, allowing redistribution of the strands among subtubes. This axial motion differs from the conventional concept of reptation because it occurs in response to stochastic (Brownian) as well as nonstochastic forces. The axial motion of the chain through entanglement junctions converts stored strain energy to thermal energy; we treat this as a dissipative frictional force acting on the strands at the entanglement junctions.

Consider a crosslinked chain constrained in an entanglement tube with an end-to-end vector $\mathbf{r}_{c}$ [Fig. 1(a)], divided by entanglement junctions into $i$ strands and subtubes with orientation $\mathbf{r}_{i}$. The end-to-end vector of the entire chain, $\mathbf{r}_{c}$, can be expressed as the sum

$$
\mathbf{r}_{c}=\sum_{i=1}^{N} \mathbf{r}_{i}
$$

of the end-to-end vectors of all strands. The number of strands $N$ in any given chain and the end-to-end strand orientation vectors $\left(\mathbf{r}_{i}\right)$ are random variables with some distribution of values. The distribution may change over time because chain motion alters the locations of the entanglement junctions along the length of the chain.

Application of an external bulk strain perturbs the subtubes' equilibrium shapes and changes the local forces acting on the strands. Each subtube's deformation depends on its orientation relative to the applied strain [Simon (1995)]. Therefore, the forces on the strands in two noncollinear consecutive subtubes will differ. The force imbalance at the entanglement junction between two noncollinear subtubes causes the chain to slide from one subtube to the next. The reallocation of the strands among adjoining subtubes relaxes the forces on the strands and creates a time-dependent viscoelastic response. Conservation of the linear momentum of all strands governs the relationship between the applied strain and the resultant stress (the product of force per strand and the number of strands per unit area) in the material. 

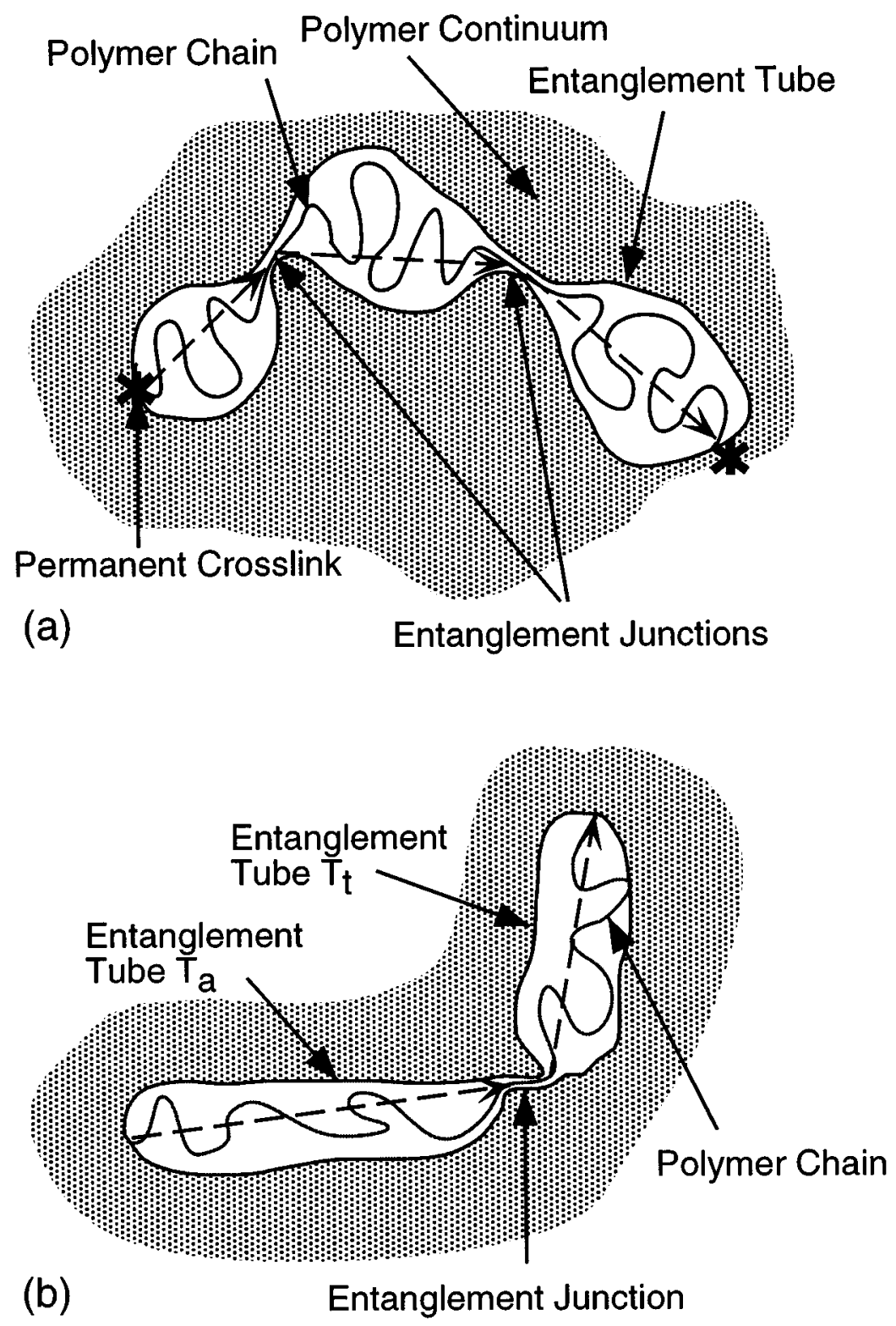

FIG. 1. Schematic representation of a typical polymer chain contained within an entanglement tube and divided into strands by entanglement junctions. The dashed arrows are the end-to-end vectors of the subtubes containing each strand. (a) General representation. (b) Two-tube approximation.

\section{B. Conservation of linear momentum}

We regard the chain as a flexible, inextensible continuous space curve that originates and terminates at crosslinks and passes through $N-1$ entanglement junctions. The control volume consists of the volume $V$ occupied by the portion of the chain within the entanglement junction. Applying the conservation of linear momentum for this control volume gives 


$$
\int_{V} \rho \frac{D \mathbf{u}}{D t} d V=\sum \mathbf{F}
$$

where $D / D t$ denotes the material derivative [Slattery (1981)], $\mathbf{u}$ is the velocity of the chain segment in $V$, and $\Sigma \mathbf{F}$ denotes the sum of all forces acting on the segment. For a chain segment of uniform density $\rho$ moving with a uniform velocity $\mathbf{u}$, Eq. (2) reduces to

$$
m \frac{D \mathbf{u}}{D t}=\sum \mathbf{F},
$$

where $m=\rho V$ is the mass of the chain segment within the control volume. Using dimensional analysis with specific force expressions (to be introduced below), we can show that the left-hand side of Eq. (3) can be neglected without any significant loss of accuracy (Appendix A), leaving

$$
\sum \mathbf{F}=0
$$

Specification of various forces, described below, make Eq. (4) a first-order ordinary differential equation governing the time dependence of chain motion through the entanglement junction.

We assume that strands are subjected to three types of forces. Expansion of a subtube increases the mean separation of the strand from neighboring chains; van der Waals attraction creates a cohesive force that pulls chain segments into the subtube to decrease the mean separation back towards the equilibrium value. Perturbation of the end-to-end distance of the strand generates a restoring elastic (entropic) force that also redistributes chain segments among the strands. A dissipative frictional force resists the motion of the chain through entanglement junctions. All of these forces will be described in more detail in the next section.

As a first approximation, we restrict our attention to a "two-tube" picture [Fig. 1(b)], which assumes only a single entanglement junction per chain and reduces the problem to that of a single relaxation-time process. Next, we ignore the distribution in subtube orientations and select a single configuration consisting of one subtube $T_{a}$ oriented coaxially with the applied elongation, and the other subtube $T_{t}$ oriented transversely to it. This configuration permits a simple model for the cohesive force and produces the largest possible force differential across the single entanglement junction. Consequently, the two-tube approximation leads to the shortest relaxation time in the response. Overall, this approximation may preclude quantitatively accurate predictions but preserves the correct qualitative features observed in experimental data.

In this analysis, we will only consider deformations corresponding to uniaxial elongation. Two factors motivate this choice. First, the molecular consequences of uniaxial elongation are easier to visualize than those of shear deformation. Second, uniaxial elongation simplifies our treatment of the entropic forces created by strand deformation. For simplicity, we also assume that the positions of crosslinked end points and entanglement junctions move affinely with the applied deformation.

\section{FORCE MODELS}

This section introduces expressions for the cohesive force, the entropic force, and the temperature-dependent frictional force acting on a representative chain. These forces, balanced in Eq. (4), are responsible for the observed features of the glassy and rubbery 
regimes and the glass transition. In order to extend the model to predict the $\beta$ transition, the next section introduces an expression for the time-dependent Poisson's ratio.

\section{A. The cohesive force and mechanical volume}

Application of an external bulk strain changes the average intermolecular separation in the material. Uniaxial extension increases the intermolecular distances in the axial direction. The cohesive force represents the net van der Waals attraction that acts on a strand to restore the equilibrium value of the average intermolecular separation. Weiner (1983) gives a rigorous statistical mechanical derivation of the cohesive force for simple molecules arranged in a lattice. No analogous derivation exists for polymers. Instead, we develop a heuristic model that relates the cohesive force to phenomenological quantities from linear elasticity theory.

Specifically, the bulk modulus quantifies the cohesive force associated with changes in the average intermolecular distances due to dilatation. In the case of uniaxial elongation in the direction of a unit vector $\mathbf{e}_{i}$, the only nonzero stress component that exists at every point in the material is

$$
\sigma_{11}=3 K \epsilon(1-2 \nu),
$$

where $K$ is the bulk modulus, $\epsilon$ is the uniaxial strain, and $\nu$ is Poisson's ratio. We postulate that the cohesive force $\mathbf{F}^{c}$ that acts on a chain segment at an entanglement junction is

$$
\mathbf{F}^{c}=\frac{\sigma_{11}}{\bar{n}} \mathbf{e}_{i}=\frac{3 K \epsilon(1-2 \nu)}{\bar{n}} \mathbf{e}_{i},
$$

where $\bar{n}$ is the number of axial subtubes or strands per unit area. Since the Poisson's ratio of the bulk material corresponds to the ratio of strains in different directions, we use the bulk value to describe the deformation of the axial subtube. Thus, in Eq. (6), $\epsilon$ and $\nu$ are the uniaxial deformation and the local Poisson's ratio, respectively, associated with a given subtube. Equation (6) and the definition of $\nu$ imply that the magnitude of the cohesive force per junction for this deformation,

$$
\left|\mathbf{F}^{c}\right|=\frac{3 K \epsilon(1-2 \nu)}{\bar{n}}=\frac{3 K}{\bar{n}} \frac{\Delta V}{V},
$$

is proportional to the local dilatation $\Delta V / V$ a force constant equal to $3 K / \bar{n}$. By analogy with thermal-free volume induced by temperature changes, we call the deformationinduced dilatational volume, $\Delta V$, the mechanical volume. The mechanical volume $\Delta V$ in the axial subtube provides the driving force for reptation of the chain into subtube $T_{a}$.

Uniaxial elongation has different effects on strands and subtubes oriented coaxial and transverse to the elongation. Elongation of the axial subtube $T_{a}$ creates mechanical volume within $T_{a}$ and an axial cohesive force that promotes reptation of the chain from $T_{t}$ through the entanglement junction into $T_{a}$. Elongation of the transverse subtube $T_{t}$ creates mechanical volume in $T_{t}$, but the associated cohesive force acts transverse to the strand and has zero resultant due to symmetry. Equation (4), therefore, includes a cohesive force term only for the part of the chain in $T_{a}$.

Two relaxation mechanisms can reduce the mechanical volume and the corresponding driving force for chain reptation. First, molecular motion of neighboring chains may decrease the intermolecular separation and relax some of the initial strain in the subtubes. Rapid side-chain and backbone bond rotations lead to $\beta$ relaxations [Arridge (1975)] 
observed at low temperatures or short time scales. The next section will explain our phenomenological approach that incorporates the $\beta$ relaxation and other secondary relaxations in our model.

Second, chain reptation into $T_{a}$, decreases the average intermolecular separation between the strand and the tube walls and, consequently, reduces the mechanical volume and the corresponding cohesive force. We assume that the decrease in mechanical volume is proportional to the volume of the chain reptating into the subtube. Let $\nu_{0}$ be the equilibrium tube volume per unit length of chain and $l_{0}$ be the initial length of strand in tube $T_{a}$. The equilibrium volume $V_{0}$ of the tube $T_{a}$ is approximately $\nu_{0} l_{0}$. In time $t$ from the application of the strain, a length of chain $x(t)$ enters the subtube $T_{a}$. Thus, the mechanical volume decreases by $\nu_{0} x(t)$ and

$$
\Delta V(t)=V_{0} \epsilon[1-2 \nu(t)]-\nu_{0} x(t)
$$

represents the variation of the total mechanical volume with time. The mechanical volume fraction relative to the original volume is then

$$
\frac{\Delta V(t)}{V_{0}}=\epsilon[1-2 \nu(t)]-\frac{x(t)}{l_{0}} .
$$

Substituting Eq. (9) into Eq. (7) gives the magnitude of the cohesive force

$$
\mathbf{F}^{c}(t)=\frac{3 K}{\bar{n}}\left\{\epsilon[1-2 \nu(t)]-\frac{x(t)}{l_{0}}\right\},
$$

as a function of the reptation length $x(t)$, the time-invariant glassy bulk modulus $K$, and the time-dependent Poisson's ratio $\nu(t)$.

The measured Poisson's ratio of polymers depends on the time scale of the measurement [Ferry (1980); Rigby (1967)]. Reported values for the Poisson's ratio of polymers range from 0.3 for glassy polymers to 0.5 for rubber. Although the variation appears to be small, the value has a significant influence on the calculation of other moduli because $[1-2 \nu(t)]$ varies by orders of magnitude for small changes in $\nu(t)$ when it is close to 0.5 . We, therefore, treat the Poisson's ratio as a time-dependent parameter. This provides a convenient way to include the physics associated with glassy relaxations, including the $\beta$ transition created by rapid, local molecular motion.

\section{B. Entropic force}

As a representative chain reptates through the entanglement junctions along its contour, the contour lengths of the constituent strands change. Variations in strand contour length between the fixed end-to-end distances separating junctions creates entropic forces in the strands. We employ rubber elasticity theory to derive an appropriate expression for the entropic force.

There are a number of molecular models for rubber elasticity. The Wall-Flory model [Hill (1986)] and the James and Guth (1943) phantom chain model assume that chains are unimpeded by the presence of surrounding chains. The constrained junction model [Erman and Flory (1978)], the multitube model [Gaylord (1979)], the slip-link model [Ball et al. (1981)], and hoop model [Adolf (1988)] account for the entanglement effect of neighboring polymer chains. The entanglement tubes in the present model are related to concepts from multitube models, while the entanglement junctions are related to the slip links and hoops from previous models. However,the exact mechanism by which slip links, hoops, or entanglement junctions impose topological constraints on the total chain 
is irrelevant to computing the entropic force because we are solely concerned with the chain conformations between two junctions.

Although the multitube model would be appropriate for strands in entanglement tubes, we essentially ignore the presence of the entanglement tubes by employing the simpler Wall-Flory model to derive the entropic force. At high temperatures and long times, the entropic force dominates the response; the constraints imposed by the entanglement tube are secondary, since the neighboring chains also undergo significant thermal motion. This justifies the use of the Wall-Flory model and equilibrium statistical mechanics to derive a force to be used in a dynamic model.

Entropic force expressions available in the literature usually compute the entropy change for extending the chain end-to-end distance by a factor $\alpha$. These expressions are independent of the actual contour length of the chain. However, we are interested in the entropy change associated with altering the contour length of the chains while holding the end points at fixed positions. Suppose that a strand of length $l_{0}$ between two entanglement junctions is subjected to an extension $\alpha$. Holding the end point positions fixed, chain reptation may change the strand length to $l$, corresponding to a new effective $\alpha^{\prime}$. We adapt the Wall-Flory model (see Appendix B) to compute the entropy change for this process.

We define the entropic force $F_{l}^{s}$ (in magnitude) such that $F_{l}^{s} d l$ equals the change in free energy to alter the length of all strands in the body by $d l$ at fixed end-to-end distance $\mathbf{r} \equiv\left\langle\left|\mathbf{r}_{i}\right|\right\rangle$. The internal energy and the free energy are

$$
d U=T d S-P d V+F_{l}^{s} d l,
$$

and

$$
d A=-S d T-P d V+F_{l}^{S} d l,
$$

where $F_{l}^{s}$ is a function of the applied elongation and the contour length defined through

$$
F_{l}^{s}=\left(\frac{\partial A}{\partial l}\right)_{T, V, r}=\left(\frac{\partial U}{\partial l}\right)_{T, V, r}-T\left(\frac{\partial S}{\partial l}\right)_{T, V, r} .
$$

The two terms on the right-hand side of Eq. (13) are contributions from intermolecular interactions and the strand configurational entropy. Under the assumptions of rubber elasticity, the former are much smaller than the latter. Therefore, we have

$$
F_{l}^{s} \approx-T\left(\frac{\partial S}{\partial l}\right)_{T, V, r}
$$

as an approximate expression for the entropic force.

In Appendix B, we develop an expression for $(\partial S / \partial l)_{T, V, r}$ based on Wall's theory [Hill (1986)]. This expression and Eq. (14) give

$$
F_{l}^{s}=-\frac{k T}{l_{0}}\left[\frac{1}{2}\left(\alpha^{2}-\frac{1}{\alpha}\right)-\frac{x(t)}{l_{0}}\left(\alpha^{2}-\frac{1}{4 \alpha}\right)\right],
$$

where $\alpha=1+\epsilon$ is the elongation resulting from the strain applied at the end points. Equation (15) represents a retractive (negative in magnitude) force upon increasing the strand end-to-end distance due to increasing $\alpha$ or decreasing $x(t)$ through the movement 
of the chain. Later, we will adapt this expression to describe the entropic force acting through strands in both axial and transverse tubes.

\section{Frictional force}

We assume that the frictional force, $\mathbf{F}^{\nu}(\mathbf{u})$, exerted on the chain is linearly proportional to the velocity of the chain passing through the junction. The magnitude of the force,

$$
\mathbf{F}^{\nu}[\mathbf{u}(t)]=-\zeta \mathbf{u}(t)=-\zeta \frac{d \mathbf{x}(t)}{d t}
$$

includes a negative sign to indicate that the force acts on the chain segment in the direction opposite to that of its motion. The frictional coefficient $\zeta$ is identical to the hydrodynamic friction coefficient found in both the Rouse model and the reptation model [Doi and Edwards (1986)]. Ferry (1980) describes $\zeta$ as "the average force per monomer unit required for a chain segment to push its way through its local surroundings at unit velocity.',

Ferry (1980) lists experimental values for $\zeta$ at different temperatures. These data show that $\zeta$ depends strongly on temperature [Larson (1988)]. However, we do not know of any mechanistic or molecular-level models that propose or employ a relationship between $\zeta$ and $T$. We employ a phenomenological expression based on the scaling relationship between the shift factor $a_{T}$ and $\zeta$ [Ferry (1980)], the Williams-Landel-Ferry (WLF) equation relating $a_{T}$, and $T$, and the free-volume interpretation of the WLF equation using the Doolittle expression [Doolittle (1951)]. Combining these concepts yields

$$
\zeta=\zeta_{0} \exp \left(\frac{b}{f_{\nu}}\right),
$$

where $f_{\nu}$ is the free-volume fraction within the entanglement junction, and $\zeta_{0}$ and $b$ are empirical parameters. The magnitude and temperature dependence of $f_{\nu}$ must now be specified.

The WLF model usually evaluates $f_{\nu}$ by fitting the WLF equation to experimental $a_{T}$ data. Although the resultant value, typically about 0.025 at the dilatational $T_{g}$, is essentially identical for different polymers, it cannot be readily interpreted in terms of other established free-volume concepts [Haward (1973)]. The expansion free-volume fraction, related to the excess volume in the material above its $0 \mathrm{~K}$ volume, has a value of about 0.13 . Because dilatometry provides a precise physical interpretation of free volume, we choose to interpret $f_{\nu}$ in terms of the expansion free-volume fraction. This interpretation establishes a vital relationship between dynamic properties (specifically, $\zeta$ ) and the thermodynamic state of the material (via the specific volume).

The temperature dependence of the expansion free-volume fraction $f_{\nu}$ may be obtained directly from dilatometric measurements. All of the qualitative features of the viscoelastic behavior predicted by the model, presented in the next section, can be obtained by using a simple linear relationship between the free-volume fraction and temperature

$$
f_{\nu}=f_{\nu 0}+\alpha_{g} T
$$

where $\alpha_{g}$ is the thermal expansion coefficient of the expansion free volume and $f_{\nu 0}$ is the free-volume fraction at $0 \mathrm{~K}$. We employ a more accurate representation of dilatometric data 


$$
f_{\nu}=f_{\nu 0}+\alpha_{g} T+\left(\alpha_{r}-\alpha_{g}\right)\left(T-T_{d g}\right) H\left(T-T_{d g}\right),
$$

that includes a dilatational glass transition at a temperature $T_{d g}$ above which the thermal expansion coefficient increases to a rubbery value $\alpha_{r} ; H(\cdot)$ is the Heaviside step function.

\section{TRANSITIONS IN THE GLASSY STATE}

Local molecular motions, such as vibrations and rotations of side groups, dissipate mechanical energy [Arridge (1975)]. This dissipation can be observed as a loss tangent peak in dynamic mechanical spectra known as the $\beta$ transition. Because the molecular architecture of side groups controls the magnitude and characteristic time/temperature of the $\beta$ transition and other secondary transitions, our coarse-grained model of the polymer as a smooth, flexible chain cannot describe this phenomenon.

We, therefore, use a phenomenological approach that builds upon our concept of mechanical volume to incorporate the effects of local molecular motions manifested as glassy relaxations typified by the $\beta$ transition. As discussed earlier, applied strain deforms the entanglement subtubes and creates local dilatation, or mechanical volume. Because the dilatation acts against attractive van der Waals forces, the creation of mechanical volume may be viewed as the storage of mechanical energy. Local dilatation stores mechanical energy in the stretching or bending of bonds of the neighboring chains comprising the subtube.

The thermal motions of neighboring chains lead to configurations that reduce their strain energy and decrease the local mechanical volume. Because the applied strain fixes the axial elongation of the subtube, the decrease in volume occurs through lateral (Poisson) contraction in the transverse stress-free direction. Thus, the glassy relaxation corresponds to a change in the local Poisson's ratio associated with the subtube.

To account for the glassy relaxation of the neighboring chains of the subtube, Eq. (10) incorporates a local, time-dependent Poisson's ratio $\nu(t)$. We assume that the local side group rotations of neighboring chains occur much more quickly than the reptation of the strand inside the subtube. In mathematical terms, $\nu(t)$ does not depend on $x(t)$. In Eq. (10), $x(t)$ is zero on the time scale of the glassy relaxations, and $\nu(t)$ decays to some ultimate value $\nu_{\infty}$ before reptation begins. The independence of glassy state relaxations and glass transitions in isochronal temperature sweeps provide experimental support for this assumption.

The present objective is to specify $\nu(t)$. Configurational barriers to the local rotational motion of side groups on neighboring chains suggest that we treat the lateral Poisson contraction and concomitant reduction of the mechanical volume as a temperaturedependent rate process. The mechanical volume $\Delta V$ [Eq. (7)] cannot be completely filled by the configurational relaxation of neighboring chains as evidenced by observed values of the Poisson's ratio between 0.3 and 0.4 for glassy polymers. Let $\Delta V_{\infty}$ represent the portion of $\Delta V$ remaining after the glassy relaxations. We postulate that the driving force for the local side group rotations of neighboring chains is proportional to the difference between the current and ultimate values of $\Delta V$. A rate equation for the reduction of mechanical volume by local side group rotations can be written as

$$
\frac{d \Delta V(t)}{d t}=-B_{c}(T)\left[\Delta V(t)-\Delta V_{\infty}\right],
$$

where the rate constant $B_{c}(T)$ represents a temperature-dependent inverse relaxationtime constant of the process. Dividing by $V_{0}$ gives an equivalent expression, 


$$
\frac{d \phi_{m}(t)}{d t}=-B_{c}(T)\left[\phi_{m}(t)-\phi_{m}^{\infty}\right]
$$

in terms of the mechanical volume fraction $\phi_{m}(t) \equiv \Delta V(t) / V_{0}$ and its limiting value $\phi_{m}^{\infty}$. Because the reptation length $x(t)$ of the chain is essentially zero on the time scale of this relaxation, Eq. (21) is independent of $x(t)$ and can be solved separately to give

$$
\phi_{m}(t)=\phi_{m}^{\infty}+\left(\phi_{m}^{0}-\phi_{m}^{\infty}\right) \exp \left[-B_{c}(T) t\right]
$$

after using the initial condition given by $\phi_{m}(0) \equiv \phi_{m}^{0}$.

As discussed earlier, the change in the mechanical volume can be expressed in terms of the local Poisson's ratio of the subtube. Setting $x(t)$ to zero in Eq. (9),

$$
\phi_{m}(t)=\frac{\Delta V(t)}{V_{0}}=\epsilon[1-2 \nu(t)]
$$

relates the local Poisson's ratio to $\phi_{m}(t)$ with

$$
\phi_{m}^{0}=\frac{\Delta V(0)}{V_{0}}=\epsilon\left(1-2 \nu_{0}\right),
$$

initially, and

$$
\phi_{m}^{\infty}=\frac{\Delta V(\infty)}{V_{0}}=\epsilon\left(1-2 \nu_{\infty}\right)
$$

after completion of all glassy relaxations. Here, $\nu_{0}$ is the instantaneous Poisson ratio observed over a time scale faster than some or all of the molecular relaxations in neighboring chains. We can set $\nu_{0}=0$ if we account for all glassy relaxations. The other Poisson ratio, $\nu_{\infty}$, is that measured on a time scale slower than side group relaxations of neighboring chains, corresponding to a value measured at a temperature near the glass transition. Presuming that most experimental measurements of the Poisson ratio satisfy this condition, we assume that $\nu_{\infty}$ takes a value in the range from 0.35 to 0.40 .

We describe the temperature dependence of $B_{c}(T)$ using an Arrhenius form

$$
B_{c}(T)=B_{c 0} \exp \left(-\frac{\Delta H_{c}}{R T}\right),
$$

where $\Delta H_{c}$ is the activation energy for the reorientation of neighboring chain side groups. In an amorphous solid, chain configurations vary from place to place and, thus, create a distribution in $\Delta H_{c}$. Because side group rotations occur at different rates at different locations in the material, we should employ a volume-averaged mechanical volume fraction. If $\phi_{m}\left(\Delta H_{c}, t\right)$, described by Eqs. (22) and (26), is the local mechanical volume fraction associated with regions having an activation energy of $\Delta H_{c}$, then

$$
\phi_{m}\left(\Delta H_{c}, t\right) P\left(\Delta H_{c}\right) d H_{c}
$$

is the average mechanical volume fraction in material relaxing with activation energies between $\Delta H_{c}$ and $\Delta H_{c}+d H_{c} . P\left(\Delta H_{c}\right) d H_{c}$ is the fraction of the material that has regions where the activation energy for chain relaxations is between $\Delta H_{c}$ and $\Delta H_{c}$ $+d H_{c}$. Integrating over all values of the activation energy yields the average mechanical volume fraction in the material 


$$
\bar{\phi}_{m}(t)=\int_{0}^{\infty} \phi_{m}\left(\Delta H_{c}, t\right) P\left(\Delta H_{c}\right) d H_{c}
$$

as a function of time.

We now relate the average mechanical volume fraction to the time-dependent Poisson's ratio in Eq. (23). Substitution of $\phi_{m}\left(\Delta H_{c}, t\right)$ from Eq. (22), $\phi_{m}^{0}$ from Eq. (24), and $\phi_{m}^{\infty}$ from Eq. (25) into Eq. (28) gives a time-temperature dependent Poisson's ratio

$$
\nu(t)=\nu_{\infty}-\left(\nu_{\infty}-\nu_{0}\right) \int_{0}^{\infty} \exp \left[-B_{c}(T) t\right] P\left(\Delta H_{c}\right) d H_{c},
$$

where $B_{c}(T)$ is given by Eq. (26).

Equation (29) can be used in Eq. (10) to express the effect of the relaxation of neighboring chain side groups on the cohesive force. To evaluate this integral, we need to know the distribution $P\left(\Delta H_{c}\right)$ of molecular relaxations having activation energy $\Delta H_{c}$. This information may be obtained through molecular dynamics modeling or spectroscopic measurements. A suitable molecular dynamics model must provide the distribution of intramolecular energies for an isolated polymer chain as well as account for the spread of the energy distribution associated with cooperative movement of neighboring chains in all the possible local environments. No such model is currently available. Alternately, $P\left(\Delta H_{c}\right)$ could be extracted from spectroscopic measurements. Broad peaks observed in the infrared spectra of most amorphous solids are a consequence of energy dispersion due to minute variations in hinderances to molecular relaxations. The effect of different postulated functional forms of $P\left(\Delta H_{c}\right)$ on the material response will be explored in the results section.

By setting $\nu(t)=\nu_{\infty}$, we can restrict the model to predict only features associated with the glass transition. Inclusion of a time-temperature dependent Poisson's ratio extends the practical range of the model by as much as $200 \mathrm{~K}$ below $T_{g}$. This introduces the need for a distribution function for the relaxation activation energy and two new parameters, $\nu_{0}$ (here, set to 0 ) and $B_{c 0}$, to be fit by experimental data.

\section{DYNAMIC MODULUS}

Based on the free-body diagram shown in Fig. 2, Eq. (4) reduces to

$$
\mathbf{F}^{c}+\mathbf{F}_{a}^{s}+\mathbf{F}_{t}^{s}+\mathbf{F}^{\nu}=0,
$$

where $a$ and $t$ denote axial and transverse directions as specified by the two-tube model. These forces depend on $x(t)$, the strand length that has translated from $T_{t}$ into $T_{a}$. Equation (30) is a function of $x(t)$ through the cohesive force $F^{c}$ [Eq. (10)] and the entropic forces $F_{a}^{s}$ and $F_{t}^{s}$. To keep the magnitudes of $F_{a}^{s}$ and $F_{t}^{s}$ consistent with the presumed directions of all of the forces at the junctions, we require $F_{a}^{s}=-F_{l}^{s}$ and $F_{t}^{s}$

$=F_{l}^{s}$ with $F_{l}^{s}$ defined by Eq. (15). In calculating $F_{t}^{s}$, we have $\alpha \approx 1$ and $x(t)<0$. Equation (30) is also a function of $d x(t) / d t$ through the frictional force term $F_{\nu}$ [Eq. (16)]. In principle, the differential equation can be solved using initial conditions $x(0)$ $=0$.

The elongational stress can be computed from the forces acting across a hypothetical dividing plane having a unit normal in the axial (elongational) direction. Substitution of the functional form for $x(t)$ into Eqs. (10) and (15) yields the forces $F^{c}$ and $F_{a}^{s}$ in the axial strands. The elongational stress can be written as 


\section{Control Volume in Entanglement Junction}

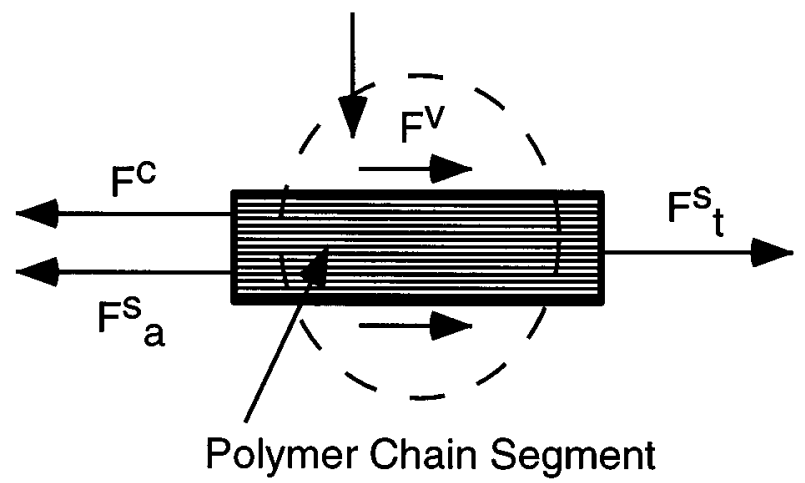

FIG. 2. Free-body diagram depicting the forces acting on a polymer chain segment within an entanglement junction.

$$
\sigma(t)=\bar{n}\left[F^{c}(t)+F_{a}^{s}(t)\right]
$$

by multiplying the total force in the axial strands by the number of strands per area, $\bar{n}$. From this, we can readily calculate the uniaxial elongation relaxation modulus $E(t)$ from

$$
E(t)=\frac{\bar{n}\left[F^{c}(t)+F_{a}^{s}(t)\right]}{\epsilon}
$$

within the framework of linear viscoelasticity.

We obtain the dynamic modulus via the sine and cosine Fourier transforms of $E(t)$

$$
E^{*}(i \omega)=i \omega \int_{0}^{\infty} E(t) \exp (-i \omega t) d t .
$$

For crosslinked polymers with moduli that do not decay to zero at $t \rightarrow \infty$, modified expressions

$$
E^{\prime}(\omega)=\omega \int_{0}^{\infty}\left[E(t)-E_{e}\right] \sin (\omega t) d t+E_{e},
$$

and

$$
E^{\prime \prime}(\omega)=\omega \int_{0}^{\infty}\left[E(t)-E_{e}\right] \cos (\omega t) d t
$$

must be used [Ferry (1980)].

Alternatively, we can obtain the dynamic modulus through a much simpler route. The integral in Eq. (33) is similar to the Laplace transform of $E(t)$ with $i \omega$ as the transform variable. We can, therefore, directly calculate the dynamic modulus by taking the Laplace transforms of Eqs. (30) and (32) to obtain

$$
E^{*}(i \omega)=\lim _{p \rightarrow i \omega} p \bar{E}(p)=\lim _{p \rightarrow i \omega} p \frac{\bar{n}\left[\bar{F}^{c}(p)+\bar{F}_{a}^{s}(p)\right]}{\epsilon},
$$


in terms of the transform variable $p=i \omega$. This procedure, described in more detail elsewhere [Simon (1995)], drastically simplifies the solution process. First, the differential equation becomes an algebraic equation in the Laplace domain. Second, we avoid the integrations described above to obtain the dynamic moduli. The procedure reduces calculation time as well as computation errors associated with numerical differentiation and integration.

The uniaxial elongation modulus can be used in conjunction with the bulk modulus to calculate other time-dependent moduli, such as shear moduli, using the elasticviscoelastic correspondence principle [Schapery (1967)]. This is possible because the bulk modulus is not a strong function of the time scale of the applied deformation. It varies roughly by a factor of 2 from the glassy to rubbery regimes, a small change compared to the two orders of magnitude observed in other moduli. We can calculate the complex shear modulus $G^{*}$ from

$$
\frac{1}{G^{*}}=\frac{3}{E^{*}}-\frac{1}{3 K}
$$

provided that we treat the bulk modulus as a constant.

\section{RESULTS AND DISCUSSION}

The model outlined in the previous section can be used to calculate the dynamic elongational and shear moduli of crosslinked polymers as functions of temperature and frequency of applied deformation. In this section, we present results for shear modulus in terms of its real and imaginary parts (storage and loss moduli). First, we discuss some of the qualitative features of this model with those of previous models. Next, we show that the calculated moduli display all of the important features observed in experimental data from temperature and frequency sweep measurements and discuss determination of model parameters and the sensitivity of predictions to parameter value choices. Finally, we present direct comparisons of calculated shear moduli with published experimental data for crosslinked epoxy resins.

\section{A. Comparison with previous theories}

The model presented here attributes the viscoelasticity of crosslinked polymers to the redistribution of the contour lengths of polymer chains within their entanglement tubes as a result of forces created by an applied deformation. Stationary entanglement junctions divide each chain into strands and the tube into subtubes. Time and temperature dependence arise from the frictional forces that oppose the sliding of chains through junctions. This model has features similar to those introduced by previous models.

Entanglement junctions resemble slip links [Ball et al. (1981)] because they constrain chain fluctuations. However, the junctions here are stationary constraints, like the hoops in the hoop model [Adolf (1988)]. Like all rubber elasticity models, we have a fit parameter, $\bar{n}$, that relates to the degree of crosslinking or the number of crosslinks. The slip-link model and the hoop model also specify the number of slip links or hoops per crosslinked chain. Our two-tube model inherently assumes a single entanglement junction per crosslinked chain. Unlike previous models, the present model employs entanglement junctions as well as entanglement tubes.

The entanglement tube concept appears in the Doi-Edwards (1986) reptation model as well as Gaylord's (1979) rubbery elasticity model. However, our model requires that various portions of the crosslinked chain reside in separate tubes. Thus, the chain strand in the subtube corresponds to a complete crosslinked chain in the Gaylord tube. Chain 
strands reptate through the tube as do uncrosslinked chains. However, the chains never leave the tube, but simply redistribute their contour length among subtubes.

The present model resembles Mooney's model (1959) in this regard because it does not require the existence of an imperfectly crosslinked network as do models based on classical reptation theory [e.g., Tsenoglou (1986)]. However, the Mooney model postulates a different mechanism for stress relaxation using the Rouse bead-spring submolecule. To compare the present model with that of Mooney, we should view a chain strand within an entanglement tube as a spring in the Mooney submolecule, and the position of an entanglement junction as that of a bead. The relaxation mechanism in our model arises from the changing length of the strands within subtubes, corresponding to changing the spring constant of the Rouse submolecule in the Mooney model. The Mooney model maintains the spring constant unchanged but assumes that the stress relaxation results from the diffusive motion of the beads within the matrix.

The true situation probably represents a combination of the two mechanisms: entanglement junctions should move as chain configurations evolve. Under these conditions, the entanglement tubes constraining the chains will also evolve. This would greatly complicate the development of the cohesive force. Despite its artificiality, our assumption that entanglement junctions deform affinely with the matrix provides the considerable simplification necessary for us to initiate our analysis.

\section{B. Qualitative behavior}

Figure 3 shows model predictions of storage $\left(G^{\prime}\right)$ and loss $\left(G^{\prime \prime}\right)$ shear moduli as functions of temperature at constant frequency of applied elongational deformation. In general, $G^{\prime}$ has high values at low temperatures, as expected for a glass. With increasing temperature, $G^{\prime}$ decreases gradually before dropping by two orders of magnitude at the glass transition. At high temperature, $G^{\prime}$ stabilizes at a rubbery plateau value. The loss modulus displays a broad peak at low temperature, representing the $\beta$ transition, as well as a sharp peak at the glass transition. As the frequency of the applied deformation decreases, the temperatures of the glass transition and $\beta$ transition shift to lower values. All of these observations correspond to features observed in experimental data from isochronal temperature sweep measurements.

The model can readily generate predictions of the frequency dependence of shear moduli at fixed values of temperature. Figure 4 shows typical $G^{\prime}$ and $G^{\prime \prime}$ results for the parameters given in Tables $I(\mathrm{a})-\mathrm{I}(\mathrm{d})$. When the applied oscillatory deformation has a high frequency, the time scale of the deformation is much smaller than all time scales of molecular motion, so $G^{\prime}$ displays high values characteristic of a glass. With decreasing frequency, the deformation time scale becomes comparable to certain kinds of molecular motion, so $G^{\prime}$ gradually decreases and $G^{\prime \prime}$ displays a $\beta$-transition peak. The time scale of polymer chain translation becomes comparable to the deformation time scale at the glass transition. Finally, at low frequency, molecular motion occurs more quickly than the applied deformation, so we observe a rubbery plateau in $G^{\prime}$ and a vanishing $G^{\prime \prime}$.

Of course, experimental time constraints preclude observation of the moduli over the complete frequency spectrum. We are typically limited to a window of frequencies delimited by the vertical lines in Fig. 4. Focusing on the $G^{\prime}$ curves in this frequency window, with increasing temperature, the curves shift downward through the glassy regime, change shape through the glass transition, and reach a rubbery plateau limit at

high temperature. The same qualitative features are observed in experimental $G^{\prime}$ data from isothermal frequency sweep measurements.

Because the model yields predictions over the entire frequency range of interest, we 


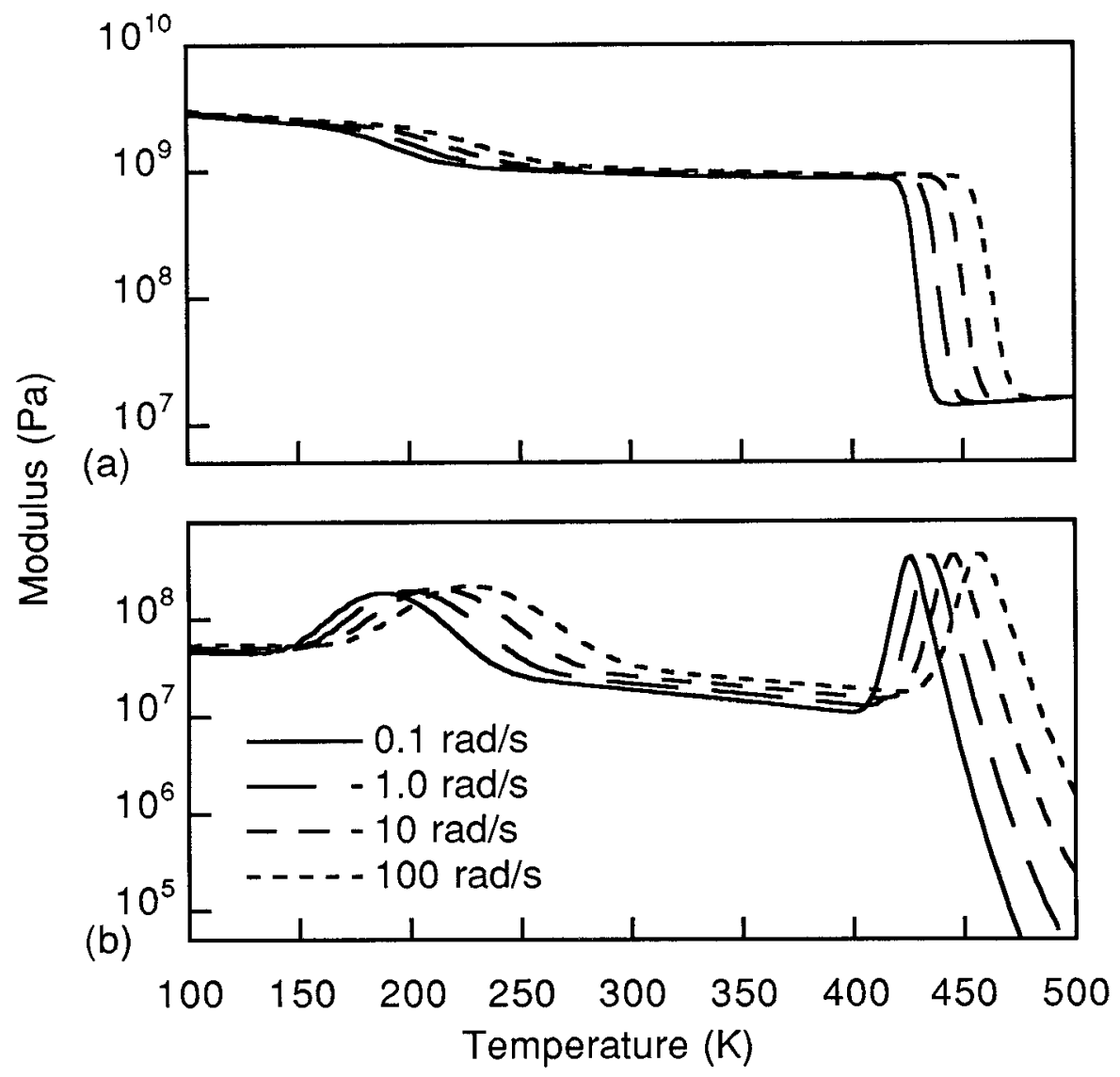

FIG. 3. Typical model predictions for $G^{\prime}$ (a) and $G^{\prime \prime}$ (b) as functions of temperature at various fixed frequencies of oscillatory elongation (parameters in Table I).

do not need to shift frequency sweeps at different temperatures over the time axis to construct a master curve (TTS). In addition, this model offers interesting possibilities for studying the validity and accuracy of TTS as applied to experimental data.

\section{Parameterization}

The tube-junction model presented here contains eight parameters listed in Tables $\mathrm{I}(\mathrm{a})$, and I(d). Of these, the four in Table I(a), $\left(K, l_{0}, \nu_{\infty}\right.$, and $\left.\nu_{0}\right)$ may be obtained from separate experiments. The four in Table $\mathrm{I}(\mathrm{d})\left(n_{m}, b, \zeta_{0}\right.$, and $\left.B_{c 0}\right)$ may be evaluated by tuning the material response so that it matches the measured dynamic mechanical data. Data from a single isochronal temperature sweep experiment are most convenient and sufficient for this purpose. We describe the evaluation of these parameters in detail in the subsequent subsections.

The model also requires two material property functions, one to describe the temperature dependence of the free-volume fraction $f_{\nu}(T)$ and the other to describe the activation energy probability distribution $P\left(\Delta H_{c}\right)$ of glassy relaxations. The first function may be obtained from thermodynamic models or experimental dilatational data. The second function may be obtained from spectroscopic measurements or molecular dynamics calculations. Lacking such data, we have used some simple functional forms to represent this 


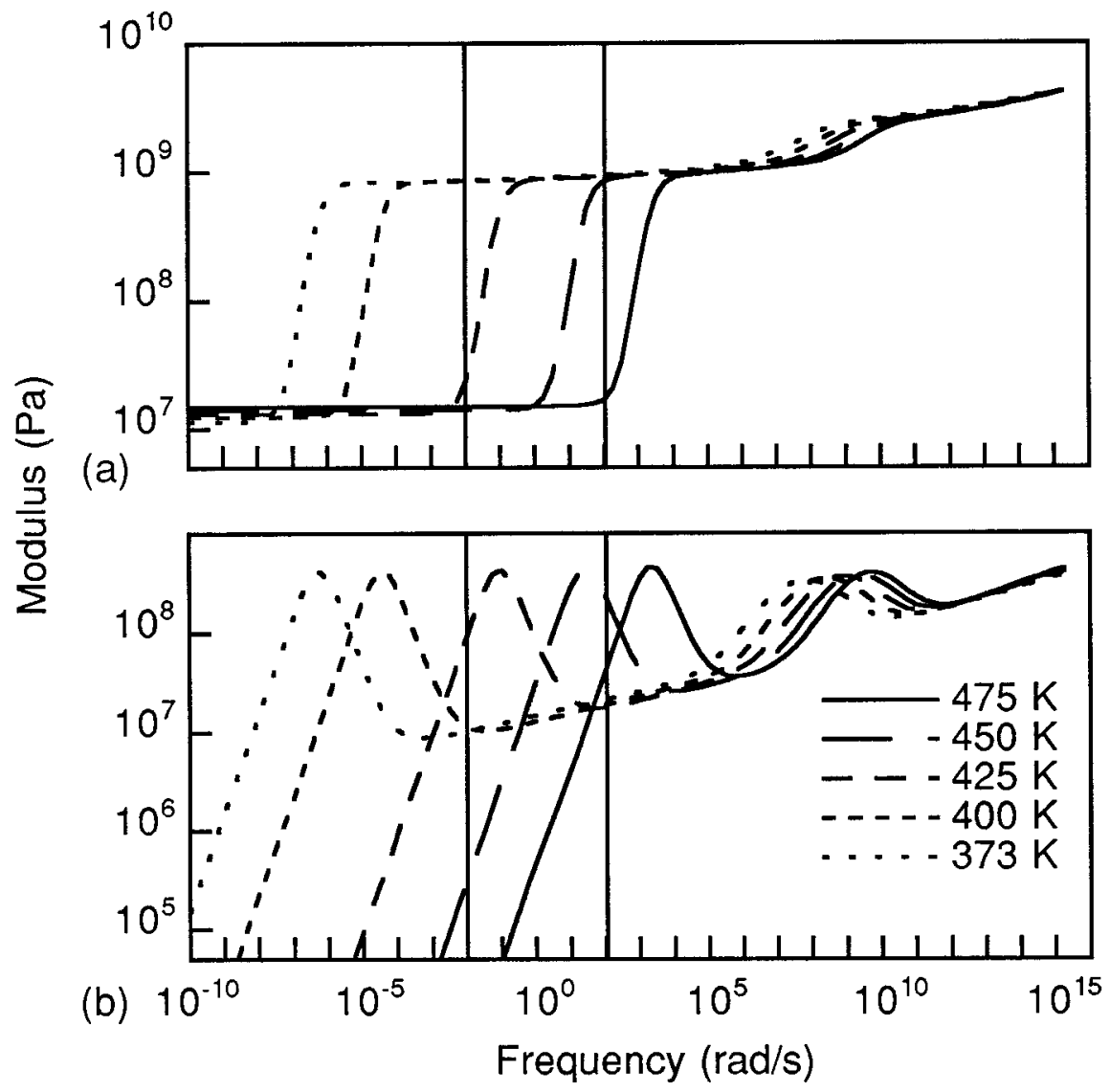

FIG. 4. Typical model predictions for $G^{\prime}$ (a) and $G^{\prime \prime}$ (b) as functions of frequency of oscillatory elongation at various fixed temperatures (parameters in Table I). The vertical lines delineate the typical window of experimentally accessible frequencies.

data. We describe below the choice of the functional forms, as well as the additional parameters associated with these functions.

\section{Material parameters}

The four parameters in Table I(a) may be determined through a variety of independent measurements. The bulk modulus $K$ varies by no more than a factor of 2 for most polymeric materials; we have selected a typical value. Knowledge of the molecular weight between crosslinks determines the strand length $l_{0}$. The molecular weight between crosslinks divided by the molecular weight per monomer equals the number of monomers between crosslinks. Multiplication of this value by the estimated contour length per monomer, divided by the number of strands per chain (two in the two-tube model) gives $l_{0}$. The value of the Poisson ratio depends on temperature or the time scale of the measurement. For glassy polymers at temperatures above the $\beta$ transition, $\nu$ $=\nu_{\infty}$ has values between 0.35 and 0.40 . This parameter can be readily measured; we have employed a typical value of 0.4 . We set $\nu_{0}=0$ in all cases, presuming that $P\left(\Delta H_{c}\right)$ spans the full activation energy spectrum. 
TABLE I. (a) Material parameters obtained from experimental measurements. (b) Free volume parameters for Eq. (19). (c) Parameters for $P\left(\Delta H_{c}\right)$, Eq. (38). (d) Parameters fitted from a selected isochrone.

\begin{tabular}{|c|c|c|}
\hline Parameter & Symbol & Value \\
\hline \multicolumn{3}{|l|}{ (a) } \\
\hline Bulk modulus & $K$ & $3.3 \times 10^{9} \mathrm{~Pa}$ \\
\hline Strand length & $l_{0}$ & $1.85 \times 10^{-9} \mathrm{~m}^{\mathrm{a}}$ \\
\hline Glassy Poisson ratio & $\nu_{\infty}$ & 0.4 \\
\hline Instantaneous Poisson ratio & $\nu_{0}$ & 0 \\
\hline \multicolumn{3}{|l|}{ (b) } \\
\hline Dilatometric glass transition temperature & $T_{d g}$ & $398 \mathrm{~K}^{\mathrm{a}}$ \\
\hline Thermal expansion coefficient below $T_{d g}$ & $\alpha_{g}$ & $1.95 \times 10^{-4} \mathrm{~cm}^{3} /\left(\mathrm{cm}^{3}{ }^{\circ} \mathrm{C}\right)^{\mathrm{a}}$ \\
\hline Thermal expansion coefficient above $T_{d g}$ & $\alpha_{r}$ & $5.70 \times 10^{-4} \mathrm{~cm}^{3} /\left(\mathrm{cm}^{3}{ }^{\circ} \mathrm{C}\right)^{\mathrm{a}}$ \\
\hline Free volume fraction at $0 \mathrm{~K}$ & $f_{v 0}$ & 0 \\
\hline \multicolumn{3}{|l|}{ (c) } \\
\hline Mean activation energy of $\beta$ transition normal distribution & $\mu_{H_{c}}$ & $63 \mathrm{~kJ} / \mathrm{mol}^{\mathrm{a}}$ \\
\hline Standard deviation of normal distribution & $\sigma_{H}$ & $6.0 \mathrm{~kJ} / \mathrm{mol}$ \\
\hline Exponential decay constant & $H^{*}$ & $40 \mathrm{~kJ} / \mathrm{mol}$ \\
\hline \multicolumn{3}{|l|}{ (d) } \\
\hline \multicolumn{3}{|l|}{ Entropic force } \\
\hline Molar number of strands per area & $n_{m}$ & $1.20 \times 10^{-4} \mathrm{~mol} / \mathrm{m}^{2}$ \\
\hline \multicolumn{3}{|l|}{ Frictional force } \\
\hline Free volume fraction parameter, Eq. (17) & $b$ & 4.0 \\
\hline Characteristic frictional coefficient, Eq. (17) & $\zeta_{0}$ & $2.0 \times 10^{-19} \mathrm{~kg} / \mathrm{s}$ \\
\hline \multicolumn{3}{|l|}{ Glassy relaxation } \\
\hline Rate constant prefactor, Eq. (26) & $B_{c 0}$ & $3 \times 10^{16} \mathrm{~s}^{-1}$ \\
\hline
\end{tabular}

a Denotes values taken from the work of Gupta et al. (1985).

\section{Temperature dependence of free volume fraction}

The temperature dependence of the free-volume fraction may be obtained from a thermodynamic equation of state or dilatational measurements. Theories describing the variation of the local free volume are subjects of current research [e.g., Cohen and Grest (1984)]. We approximated the dilatational data given by Gupta et al. (1985) with Eq. (19), a linear temperature dependence for the free-volume fraction in the glassy and rubbery thermodynamic states separated by a distinct dilatational glass transition. The four parameters used in Eq. (19) are listed in Table I(b) and are taken from Gupta et al. (1985). The value of the free-volume fraction at $0 \mathrm{~K}, f_{\nu 0}$, is assumed to be zero. The dilatometric glass transition does not directly cause the viscoelastic glass transition, although the two are indirectly related; we will explore this subject further in another publication.

\section{Activation energy probability distribution function}

The form of the activation energy distribution function $P\left(\Delta H_{c}\right)$ affects the shape of the modulus profiles in the glassy region. Figures 5, 6, and 7 show three different distribution functions and the corresponding moduli profiles. The peaks in the distribution function correspond to $\beta$ or $\delta$ relaxations. Figure 5 shows that a normal distribution of $\Delta H_{c}$ centered around the $\beta$-relaxation value yields a response unlike that observed in most polymer solids. The absence of molecular relaxation processes at higher and lower 

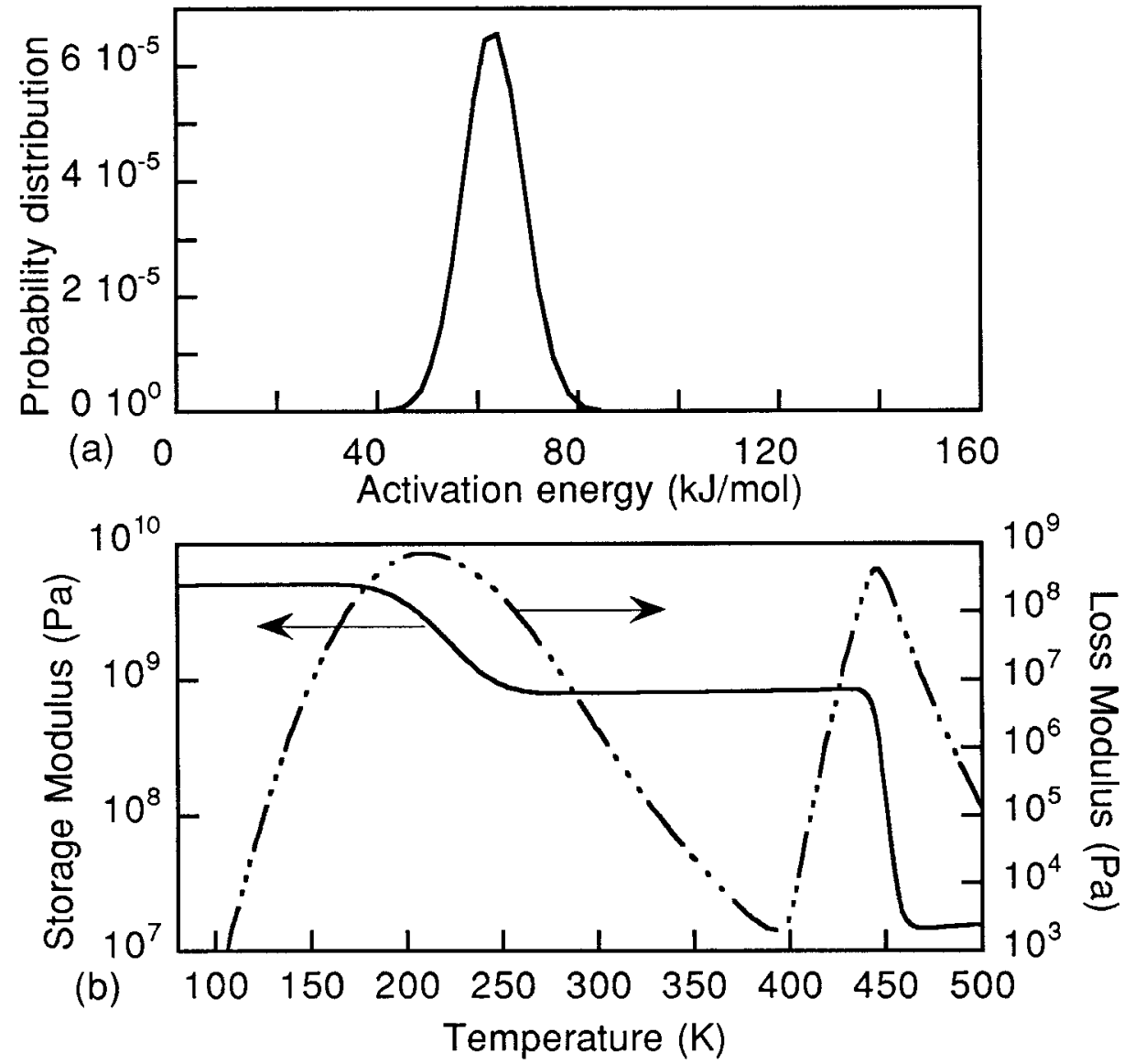

FIG. 5. (a) Normalized $P\left(\Delta H_{c}\right)$ given by a normal distribution centered on the relaxation energy of the $\beta$ transition. (b) Corresponding model predictions of $G^{\prime}$ and $G^{\prime \prime}$.

activation energies creates a deep minimum in $G^{\prime \prime}$ and a corresponding sharp step in $G^{\prime}$. Inclusion of a wider range of molecular relaxation processes, Fig. 6, shows that a broad spectrum of activation energies produces a $G^{\prime \prime}$ profile that is qualitatively similar to that observed for most crosslinked polymers. This is consistent with the expectation that molecular relaxations in solid polymers occur through the cooperative motion of neighboring chains. Figure 7 shows that an additional peak in $P\left(\Delta H_{c}\right)$ produces a corresponding peak in $G^{\prime \prime}$. In the context of this model, the activation energy distribution alone controls the shape of the transition profiles observed in the glassy regime.

It may be possible to estimate $P\left(\Delta H_{c}\right)$ by fitting the model to data from spectroscopic experiments or a detailed molecular dynamics model. Since we lack such data, we have chosen a reasonable analytical function for $P\left(\Delta H_{c}\right)$ and have optimized the function's parameters in order to match the response in the glassy region. To account for the $\beta$-relaxation peak, we assume that $P\left(\Delta H_{c}\right)$ has a normal distribution with an activation energy peak $\mu_{H c}=63 \mathrm{~kJ} / \mathrm{mol}$ [given by Gupta et al. (1985)] and a standard deviation, $\sigma_{H c}$, equal to $6.0 \mathrm{~kJ} / \mathrm{mol}$. The broader spectrum of molecular relaxations are assumed to have the form of an exponentially decaying distribution with decay constant $H^{*}$. We used 

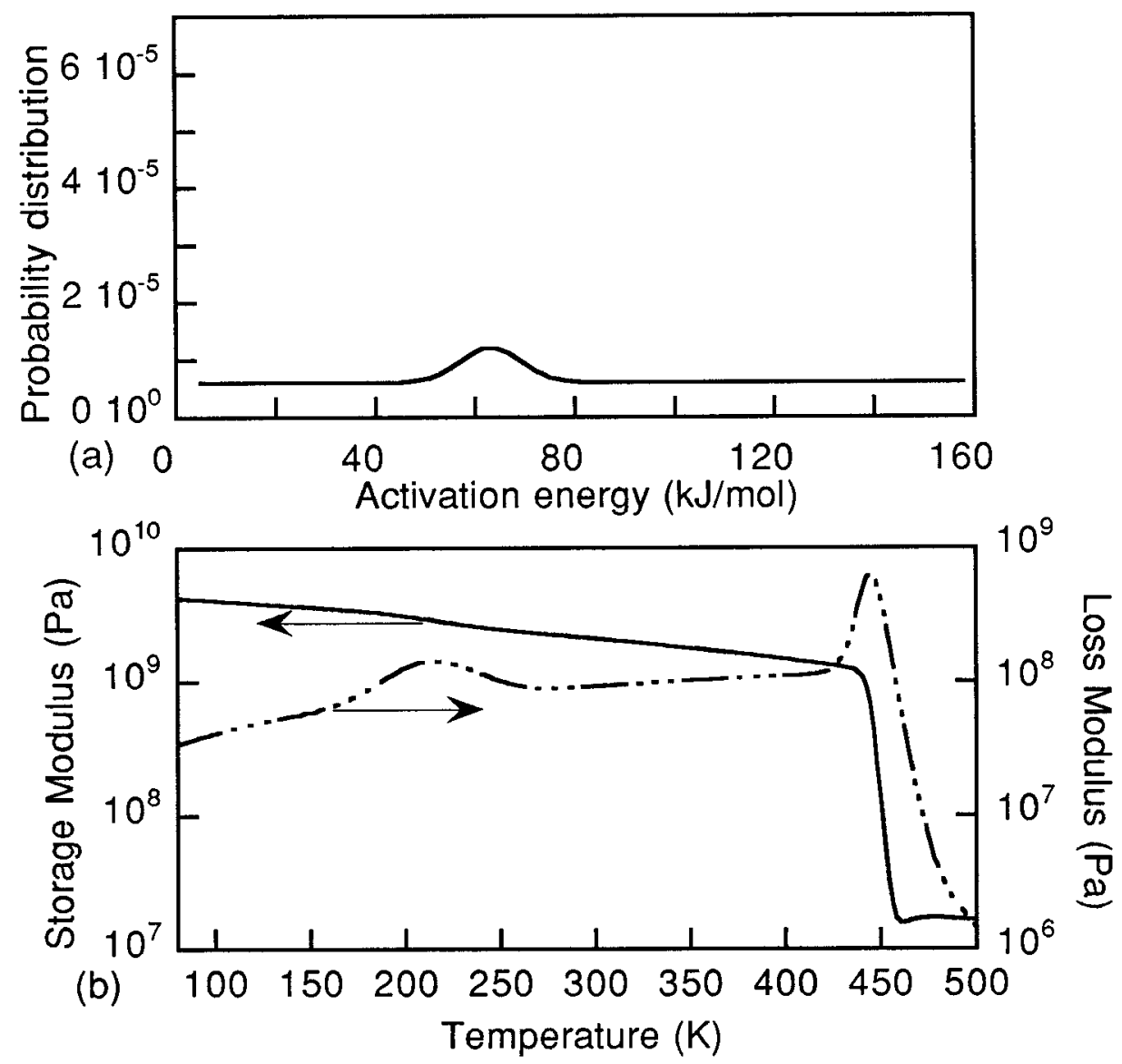

FIG. 6. Normalized $P\left(\Delta H_{c}\right)$ given by a normal distribution centered on the relaxation energy of the $\beta$ transition plus a background uniform distribution of activation energies. (b) Corresponding model predictions of $G^{\prime}$ and $G^{\prime \prime}$.

$$
P\left(\Delta H_{c}\right)=\frac{\exp \left(-\Delta H_{c} / H^{*}\right)+\exp \left[-\left(\Delta H_{c}-\mu_{H c}\right)^{2} / 2 \sigma_{H c}^{2}\right]}{H^{*}+\sqrt{2 \pi} \sigma_{H c}},
$$

with $H^{*}=40 \mathrm{~kJ} / \mathrm{mol}$. Again, these parameters only influence the features of the glassy region. Table $\mathrm{I}(\mathrm{c})$ lists the parameters used in Eq. (38).

\section{Force parameters}

The four parameters in Table I(d) appear in the definitions of the cohesive, frictional, and entropic forces developed in the previous section and must be adjusted by fitting the model to a single experimental isochrone. Below, we outline a convenient procedure for choosing values for these parameters and describe the sensitivity of the results to chosen values.

The entropic force contains one parameter, the molar number of strands per unit cross-sectional area $n_{m} \equiv \bar{n} / N_{A}$, that controls the magnitude of $G^{\prime}$ in the rubbery plateau. Figure 8 shows the effect of varying $n_{m}$ values on the predicted material response. Increasing values of $n_{m}$ shift the $G^{\prime}$ rubbery plateau to higher values and increase the 

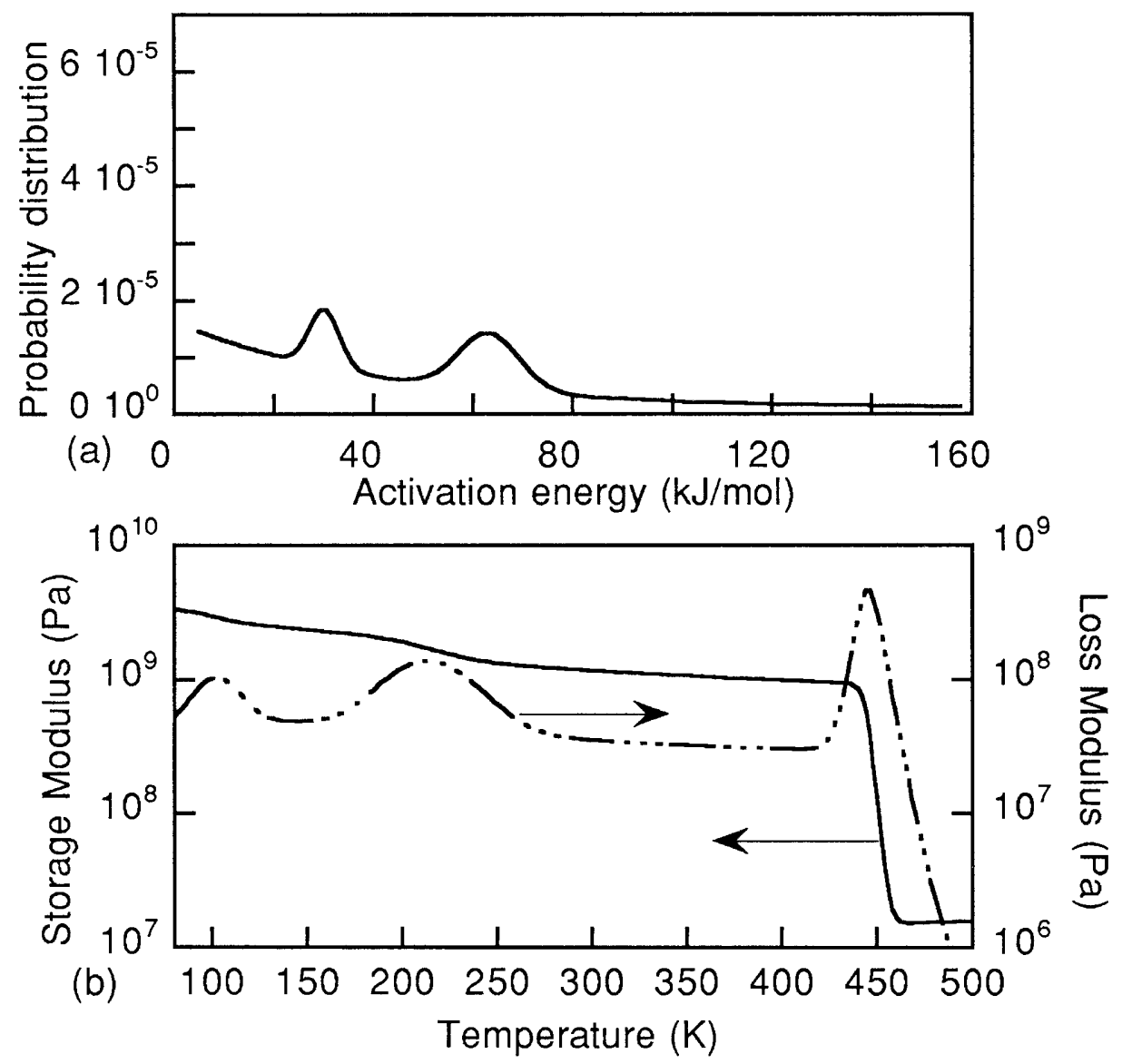

FIG. 7. Normalized $P\left(\Delta H_{c}\right)$ given by an exponentially decaying background distribution of activation energies plus two distinct normal distributions centered at 30 and $63 \mathrm{~kJ} / \mathrm{mol}$. (b) Corresponding model predictions of $G^{\prime}$ and $G^{\prime \prime}$.

viscoelastic $T_{g}$, consistent with experimental observations [Pogany (1970)]. The viscoelastic response becomes independent of $n_{m}$ at temperatures just below $T_{g}$. Thus, a measured value of $G^{\prime}$ in the rubbery plateau at a known temperature can be used to set the value of $n_{m}$, as is done in rubber elasticity models to determine the degree of crosslinking.

The frictional force introduces two parameters, $b$ and $\zeta_{0}$, in Eq. (17) relating the frictional coefficient to the thermal free volume. Figure 9(a) shows the effect of varying values of $b$ on the predicted material response. Because it appears in the exponential in Eq. (17), larger values of $b$ increase significantly the frictional coefficient and, thus, shift the glass transition to higher temperature. More importantly, larger values of $b$ reduce the slope of the storage modulus across the glass transition. This effect can also be seen in the slight decrease of the loss modulus peak with increasing $b$. The value of $b$ has no effect on the rubbery $G^{\prime}$ or any features of the response below the glass transition temperature. We choose the value of $b$ by attempting to match the predicted and experimental slopes of $G^{\prime}$ in the glass transition.

Figure 9(b) shows the effect of varying values of $\zeta_{0}$ on the predicted material re- 


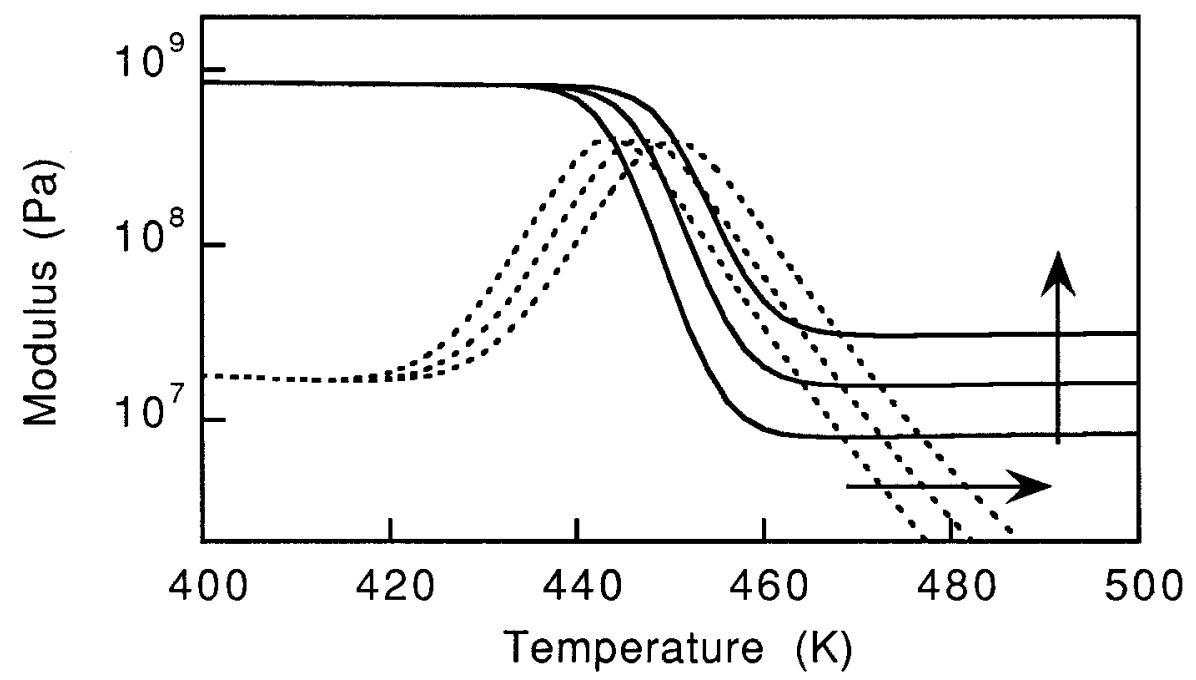

FIG. 8. Effect of varying values of $n_{m}$ on $G^{\prime}$ (solid curves) and $G^{\prime \prime}$ (dotted curves) through the glass transition and rubbery plateau. The arrows indicate the direction of increasing $n_{m}$.

sponse. Since $\zeta_{0}$ appears as a prefactor in Eq. (17), larger values of $\zeta_{0}$ increase the friction coefficient and, thus, shift the glass transition to higher temperatures. The value of $\zeta_{0}$ has little effect on the loss modulus peak and, like $b$, has no effect on the rubbery $G^{\prime}$. We can, therefore, determine the value of $\zeta_{0}$ by matching the predicted and experimental values of the temperature of the $G^{\prime \prime}$ peak at the glass transition.

Larger values of $B_{c 0}$ increase the rate constant for relaxation of side groups on neighboring chains, thus, decreasing the temperature of the $\beta$ transition (Fig. 10). We choose $B_{c 0}$ by matching the calculated and experimental values of the temperature of the $\beta$-transition peak. As discussed previously, the activation energy distribution function $P\left(\Delta H_{c}\right)$ determines the other features of the response in the glassy region.

\section{Comparison with experimental data}

The previous section outlines a systematic procedure for choosing values for the model's parameters. In Figs. 11 and 12, we compare model predictions with experimental data for cured epoxy resins measured by Gupta et al. (1985). Tables $\mathrm{I}(\mathrm{a})-\mathrm{I}(\mathrm{d})$ give the optimized set of model parameters for this comparison. The form of $P\left(\Delta H_{c}\right)$, given by Eq. (38), is similar to that shown in Fig. 7(a), but with only one peak.

Theoretical predictions and experimental data for $G^{\prime}(T)$ and $G^{\prime \prime}(T)$ are shown in Fig. 11. The chosen value of $B_{c 0}$ locates the peak of the $\beta$ relaxation at the correct temperature. The values of the frictional parameters $\zeta_{0}$ and $b$ correctly locate the $G^{\prime \prime}$ peak and the slope of $G^{\prime}$ at the glass transition. However, the model does not represent accurately the peak height of $G^{\prime \prime}$ at the glass transition.

Comparison of calculated and experimental $G^{\prime}(\omega)$ data from isothermal frequency sweep measurements [Gupta et al. (1985)] in Fig. 12 further illustrate this point. This comparison employs no additional adjustable parameters. At both low and high temperatures, the model predicts $G^{\prime}$ values close to the experimental data. We observe a significant deviation in the glass transition regime: the transition occurs over a shorter frequency span than in the experimental data. We attribute this deviation to the assumption of a single relaxation process inherent in the model's treatment of the simplified two-tube 


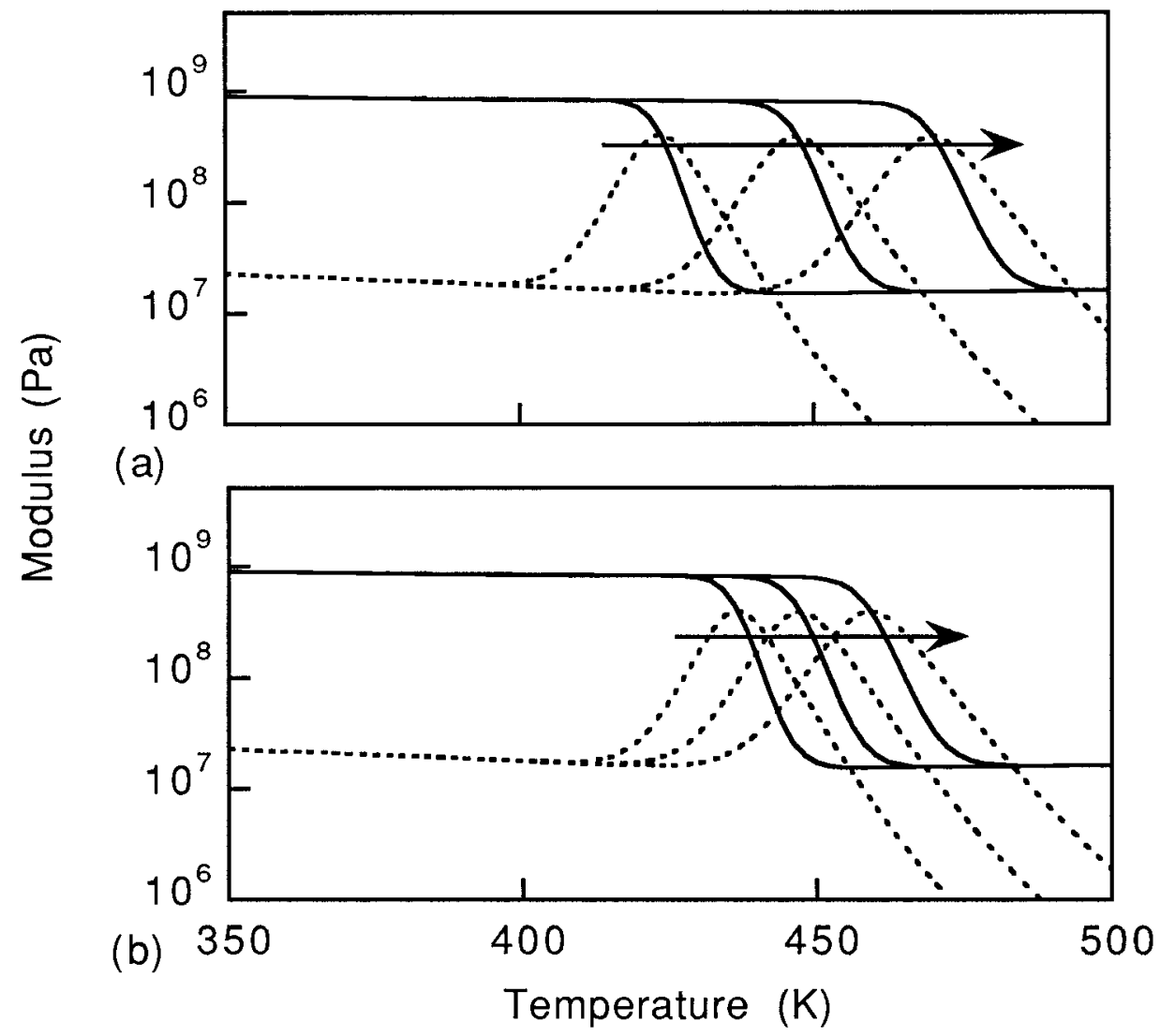

FIG. 9. Effect of varying values of $b$ (a) and $\zeta_{0}$ (b) on $G^{\prime}$ (solid curves) and $G^{\prime \prime}$ (dotted curves) at temperatures around the glass transition. The arrows indicate the direction of increasing parameter values.

configuration. The corresponding loss peak (not shown) is also larger than the corresponding experimental values. This may also explain the large loss peak in the isochrone (Fig. 11). Better prediction of material properties in the glass transition region may be obtained by incorporating a distribution of tube arrangements in a more sophisticated model.

\section{CONCLUSIONS}

In this paper, we have proposed a molecular-level description of the dynamic response of crosslinked polymers to applied uniaxial deformations. The theoretical model consists of a momentum balance on a representative segment of the polymer network as well as phenomenological descriptions of the forces acting on the segment. The cohesive force accounts for the long-range van der Waals attraction between molecules; the entropic force describes the thermodynamic effects that influence chain dimensions; and the frictional force captures the profound effect of temperature on relative chain motion. If improved force models are developed in the future, these can be used within the general framework constructed here.

The model equations can be solved in the frequency domain to calculate the dynamic moduli for applied oscillatory uniaxial elongation. Results in the form of isochronal 


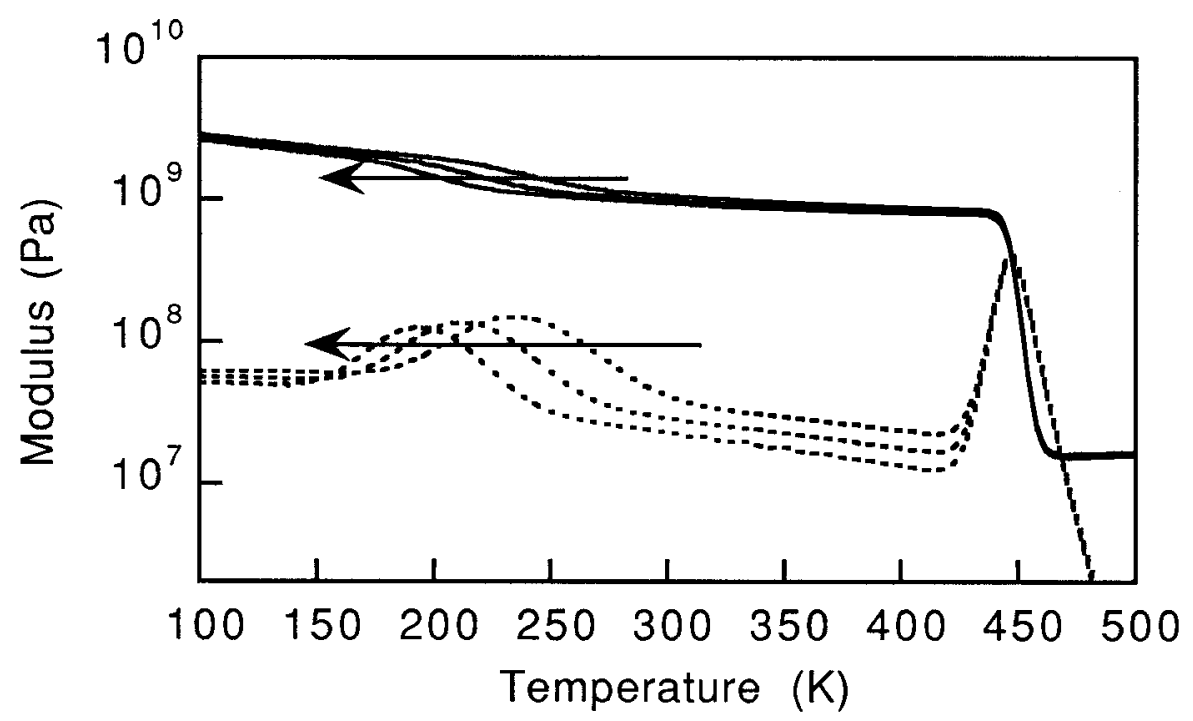

FIG. 10. Effect of varying values of $B_{c 0}$ on $G^{\prime}$ (solid curves) and $G^{\prime \prime}$ (dotted curves). The arrows indicate the direction of increasing parameter values.

temperature sweeps and isothermal frequency sweeps can be readily calculated. The model is the first, to our knowledge, that can describe the complete temperature and frequency dependence of dynamic moduli, thus, predicting the features of both $\beta$ and glass transitions.

We have described a systematic procedure for evaluating all of the model parameters through independent experimental measurements. The four adjustable parameters in the three force expressions can be determined through the use of modulus data from a single isochronal temperature sweep. We used this procedure to determine parameter values for a cured epoxy resin [Gupta et al. (1985)]. In this case, the model predicts dynamic shear moduli that agree qualitatively with experimental data. We also find quantitative agreement between theory and experiment for temperatures and frequencies outside of the glass transition region.

Although the model predicts the correct features of the dynamic moduli in the glass transition, the numerical values of the moduli are not accurate. This discrepancy may be due to approximations inherent in the two-tube form of the model: in reality, each segment of the polymer network may be contained in more than two subtubes, each having an arbitrary alignment relative to the imposed deformation. Quantitative accuracy in the glass transition zone can be improved by accounting for the distribution of subtube orientations.

The viscoelastic glass transition predicted by this model is a purely relaxational phenomenon that arises because different forces dominate in various parts of the dynamic mechanical spectrum. The viscoelastic glass transition, as described here, does not require the existance of a dilatational glass transition. However, the dilatational glass transition affects the quantitative features of the dynamic response.

In its present form, we can use the model to explore other issues related to polymer viscoelasticity. For example, the model can be used to examine the validity of timetemperature superpositioning by comparing the "complete" response (model predictions of dynamic moduli calculated over many decades of frequency) with experimental master 


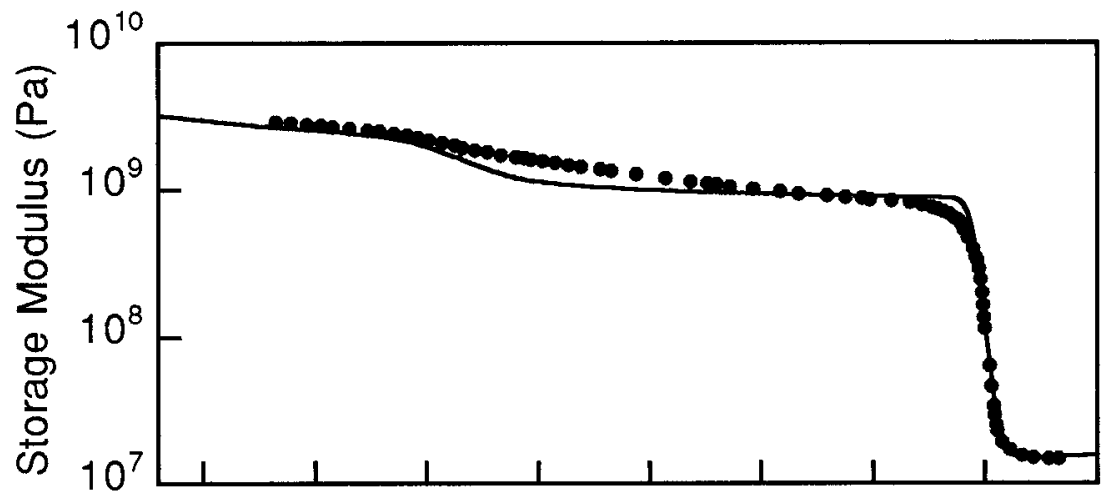

(a)

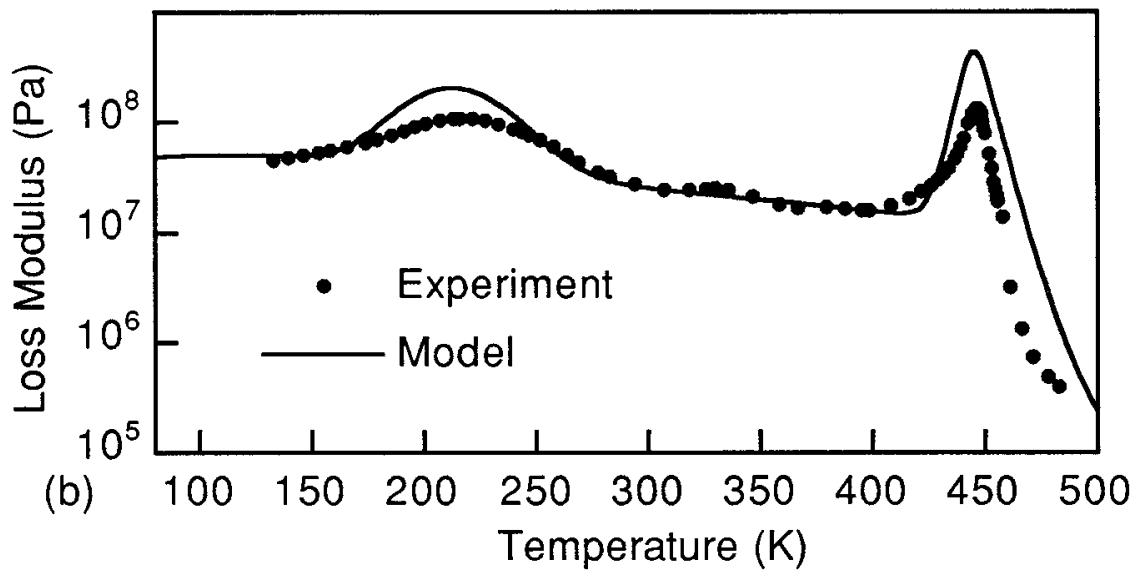

FIG. 11. Comparison of model predictions for $G^{\prime}(T)$ (a) and $G^{\prime \prime}(T)$ (b) at $\omega=10 \mathrm{rad} / \mathrm{s}$ with corresponding experimental data measured by Gupta et al. (1985). The model employs Eq. (38) for $P\left(\Delta H_{c}\right)$ and parameter values in Table I.

curves as well as "artificial" master curves constructed from calculated frequency sweeps for a limited frequency domain at different temperatures.

We believe that the combination of the molecular-level picture and the mathematical model presented here provide an interesting and useful theoretical platform for understanding polymer viscoelasticity across the complete spectrum of time and temperature.

\section{ACKNOWLEDGMENTS}

The authors wish to thank the U.S. National Science Foundation for financial support provided through Grant No. CTS-9258137 and the Offshore Technology Research Center Grant No. CDR-8721512, and the U.S. Department of Energy for support provided through Grant No. DE-FC02-91ER75666.

\section{APPENDIX A: DIMENSIONAL ANALYSIS}

We can use dimensional analysis to show that the inertial term in the momentum balance, Eq. (3), can be neglected. Using the initial strand length $l_{0}$ and introducing a characteristic time $t_{0}$, we define dimensionless translated strand length and time as $x *$ $=x / l_{0}$ and $t^{*}=t / t_{0}$. Substitution of these definitions into the left-hand side of Eq. (3) 


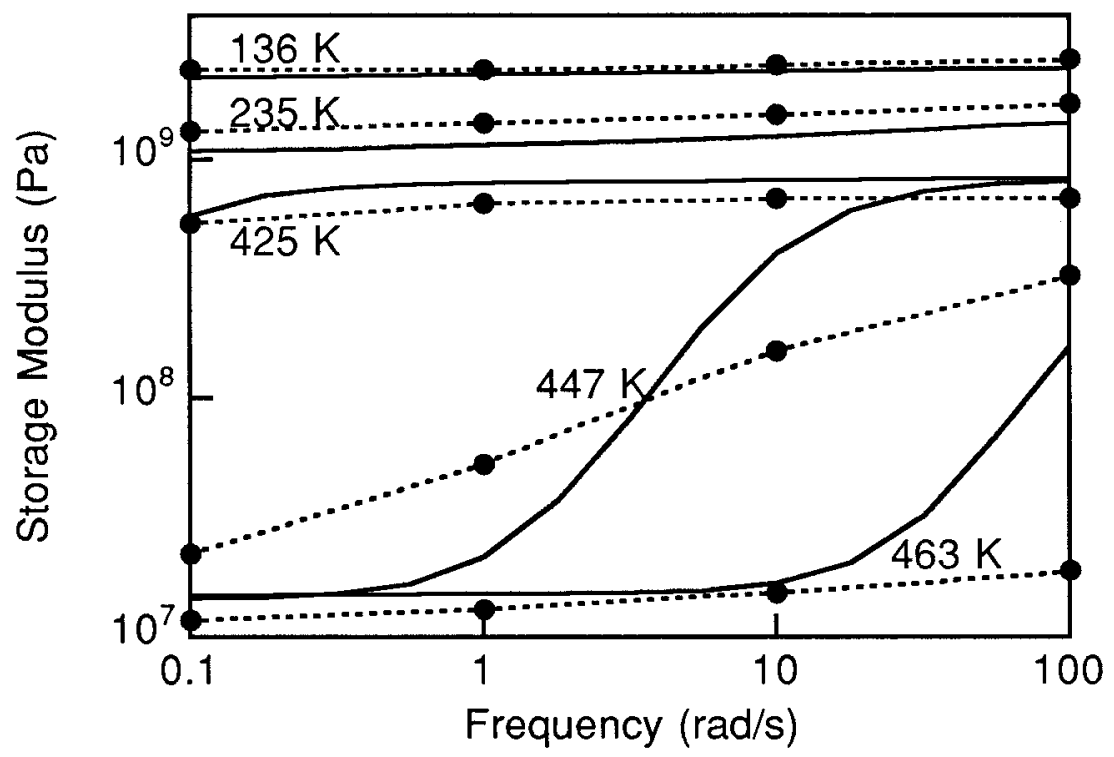

FIG. 12. Comparison of model predictions for $G^{\prime}(\omega)$ at various temperatures (full curves) with corresponding experimental data (filled circles with dotted curves) measured by Gupta et al. (1985). The model employs Eq. (38) for $P\left(\Delta H_{c}\right)$ and parameter values in Table I.

identifies the characteristic inertial force shown in Table II. Similar characteristic groups, also shown in Table II, may be identified for the cohesive and entropic forces acting on chain segments.

Using the values in Table I, as well as some additional parameter estimates, we can evaluate the magnitudes of these forces in the glassy and rubbery regimes. We choose $m=10^{-25} \mathrm{~kg}, \epsilon=10^{-4}, \nu=0.2$, and $T=475 \mathrm{~K}$. At this temperature, Fig. 4 suggests that glassy behavior would be observed for $\omega$ above about $10^{4} \mathrm{rad} / \mathrm{s}$ or $t_{0}$ $\approx 10^{-4} \mathrm{~s}$. Under these conditions, the values in Table II show that the inertial force has a much smaller magnitude than the dominant cohesive force and can, therefore, be neglected. The frictional force (not computed) would have a similar magnitude. Higher frequency would decrease $t_{0}$ and increase the characteristic inertial force relative to the cohesive force. However, the inertial force in Table II represents an upper limit since it represents the inertia of an entire strand rather than just the portion in the entanglement junction.

TABLE II. Order-of-magnitude estimates of forces in Eq. (3).

\begin{tabular}{lcccc}
\hline \hline Force & Equation & Characteristic group & Value at $t_{0}=10^{-4} \mathrm{~s}$ & Value at $t_{0}=0.1 \mathrm{~s}$ \\
\hline Inertial & $(3)$ & $\frac{m l_{0}}{t_{0}^{2}}$ & $1.9 \times 10^{-26} \mathrm{~N}$ & $1.9 \times 10^{-32} \mathrm{~N}$ \\
Cohesive & $(10)$ & $\frac{3 K}{\bar{n}} \epsilon(1-2 \nu)$ & $1.4 \times 10^{-14} \mathrm{~N}$ & $\approx O\left(10^{-20}\right) \mathrm{N}$ \\
Entropic & $(15)$ & $\frac{k T}{2 l_{0}}\left(\alpha^{2}-\frac{1}{\alpha}\right)$ & $5.3 \times 10^{-16} \mathrm{~N}$ & $5.3 \times 10^{-16} \mathrm{~N}$ \\
& & & \\
\hline \hline
\end{tabular}


At the same temperature, Fig. 4 implies that rubbery behavior would be observed for $\omega$ below about $10 \mathrm{rad} / \mathrm{s}$ or $t_{0} \approx 0.1 \mathrm{~s}$. We expect that the cohesive force has a magnitude comparable to the entropic force, although we cannot estimate the exact value since Eq. (10) depends on a finite length of strand translation. The inertial force is clearly small compared to the entropic force and can be neglected under these conditions.

Under some conditions, the inertial term in Eq. (3) may not be negligible. Retaining this term increases the order of the differential equation to be solved, but otherwise introduces no mathematical difficulties. We have performed calculations in which we have retained the inertial term and have found that it has little effect on either the qualitative or quantitative features of the results. We have, therefore, neglected this term for computational expedience.

\section{APPENDIX B: STATISTICAL THEORY FOR THE ENTROPIC FORCE}

Consider a set (I) of $N$ chains, each with length $l_{0}$, crosslinked, and in their unperturbed equilibrium state (state $A$ ). Their end-to-end distances have the most probable distribution. Upon deformation of the network (state $D$ ), the end points of the chains are stretched affinely by some extension $\alpha_{x}, \alpha_{y}, \alpha_{z}$ relative to state $A$. In order to calculate the entropy change due to change in the chain lengths and the corresponding effective change in the applied extensions, we need to consider another set (II) of chains of lengths $l$ that have the same distribution of end-to-end distances as in state $D$. Set II chains must be deformed from their most probable distribution in order to occupy the distribution in state $D$. However, it is not necessarily possible to specify a macroscopic deformation in three variables $\alpha_{x}^{\prime}, \alpha_{y}^{\prime}, \alpha_{z}^{\prime}$ that will meet this condition. In order to make further progress, we restrict our analysis to the special case of the uniaxial elongation and introduce some simplifying assumptions.

First, we assume that the elongation in the mean-square end-to-end distance approximates the elongation in the mean square of its axial (say $x$ ) component, represented by

$$
\frac{\left\langle r^{2}\right\rangle}{\left\langle r_{0}^{2}\right\rangle} \approx \frac{\left\langle x^{2}\right\rangle}{\left\langle x_{0}^{2}\right\rangle}=\alpha^{2},
$$

where $\left\langle r^{2}\right\rangle$ and $\left\langle x^{2}\right\rangle$ are the mean square of the end-to-end distance and its $x$ component, respectively, and subscript (0) denotes the unperturbed state. Second, we assume that it is sufficient for $\left\langle x^{2}\right\rangle$ in the two sets to be equal and, thereby, relax the requirement on the distribution imposed earlier. We can apply Eq. (B1) to the two sets to get

$$
\frac{\left\langle x_{0 \mathrm{II}}^{2}\right\rangle}{\left\langle x_{0 \mathrm{II}}^{2}\right\rangle}=\left(\frac{\alpha^{\prime}}{\alpha}\right)^{2},
$$

where subscripts (I) and (II) represent the two chain sets. The unperturbed mean-square end-to-end distance is proportional to the contour length of the chain [Flory (1953)] so that

$$
\frac{\left\langle r_{0 \mathrm{I}}^{2}\right\rangle}{\left\langle r_{0 \mathrm{II}}^{2}\right\rangle}=\frac{l_{0}}{l} .
$$

Equations (B1), (B2), and (B3) give

$$
\frac{l_{0}}{l}=\left(\frac{\alpha^{\prime}}{\alpha}\right)^{2}
$$


which relates the extension $\alpha$ applied to the chain ends of set I to the effective extension applied to the chain ends of set II in terms of their contour lengths.

The entropy of chain set II [Hill (1986)],

$$
\Delta S=\frac{k N}{2}\left(3-\alpha^{\prime 2}-\frac{2}{\alpha^{\prime}}\right)
$$

relative to its unperturbed state can be related to the conditions in set I through

$$
\Delta S=\frac{k N}{2}\left(3-\alpha^{2} \frac{l_{0}}{l}-\frac{2}{\alpha} \sqrt{\frac{l}{l_{0}}}\right)
$$

by substituting from Eq. (B4) for $\alpha^{\prime}$. The force defined by Eq. (14)

$$
F_{l}^{s} \approx-T\left(\frac{\partial S}{\partial l}\right)_{T, V, r}=-\frac{k T N}{2}\left(\alpha^{2} \frac{l_{0}}{l^{2}}-\frac{1}{\alpha \sqrt{l_{0} l}}\right),
$$

is a function of both the applied elongation $\alpha$ and the current length of the chain $l(t)$. The new length $l(t)$ is related to the chain displacement $x(t)$ through

$$
l(t)=l_{0}+x(t) .
$$

For small values of $x(t)$, we can use the linearized form given by Eq. (15).

\section{References}

Adams, D. F. and A. K. Miller, "Hygrothermal Microstresses in a Unidirectional Composite Exhibiting Inelastic Material Behavior,' J. Compos. Mater. 11, 285-299 (1977).

Adolf, D. "Junction Fluctuations in Confined Chain Models of Rubber Elasticity," Macromolecules 21, 22492253 (1988).

Arridge, R. G. C., Mechanics of Polymers (Oxford University Press, London, 1975).

Ball, R. C., M. Doi, S. F. Edwards, and M. Warner, "Elasticity of Entangled Networks," Polymer 22, 10101018 (1981).

Brinson, L. C. and W. G. Knauss, “Thermorheologically Complex Behavior of Multi-Phase Viscoelastic Materials," J. Mech. Phys. Solids 39, 859-880 (1991).

Cohen, M. L. and G. S. Grest, “The Nature of the Glass Transition,” J. Non-Cryst. Solids 61, 749-760 (1984).

Crossman, F. W., R. E. Mauri, and W. J. Warren, "Moisture-Altered Viscoelastic Response of Graphite/Epoxy Composites," in Advanced Composite Materials-Environmental Effects, ASTM STP 658, edited by J. R. Vinson (American Society for Testing and Materials, Philadelphia, PA, 1978), pp. 205-220.

Doi, M. and S. F. Edwards, The Theory of Polymer Dynamics (Oxford University Press, Oxford, 1986).

Doolittle, A. K., "Studies in Newtonian Flow. II. The Dependence of the Viscosity of Liquids on Free-Space," J. Appl. Phys. 22, 1471-1475 (1951).

Erman, B. and P. J. Flory, “Theory of Elasticity of Polymer Networks. II. The Effect of Geometric Constraints on Junctions," J. Chem. Phys. 68, 5363-5369 (1978).

Ferry, J. D., Viscoelastic Properties of Polymers (Wiley, New York, 1980), pp. 332 and 445.

Flory, P. J., Principles of Polymer Chemistry (Cornell University Press, Ithaca, NY, 1953).

Gaylord, R. J., "Entanglement and Excluded Volume Effects in Rubber Elasticity," Polym. Eng. Sci. 19, 263-266 (1979).

Gupta, V. B., L. T. Drzal, C. Y.-C. Lee, and M. J. Rich, "The Effects of Stoichiometry and Structure on the Dynamic Torsional Properties of a Cured Epoxy Resin System,"' J. Macromol. Sci. Phys. B 23, 435-466 (1985).

Haward, R. N., "Introduction-The Nature of Polymer Glasses, Their Packing Density and Mechanical Behavior," in The Physics of Glassy Polymers, edited by R. N. Haward (Wiley, New York, 1973).

Hill, T. L., An Introduction to Statistical Thermodynamics (Dover, New York, 1986).

James, H. M. and E. Guth, “Theory of the Elastic Properties of Rubber,' J. Chem. Phys. 11, 455-481 (1943). 
Kirkwood, J. G., "Elastic Loss and Relaxation Times in Crosslinked Polymers," J. Chem. Phys. 14, 51-56 (1946).

Larson, R. G., Constitutive Equations for Polymer Melts and Solutions (Butterworths, Boston, MA, 1988), p. 53.

Miyase, A., A. W.-L. Chen, P. H. Geil, and S. S. Wang, "Anelastic Deformation of a Thermoplastic-Matrix Fiber Composite at Elevated Temperature; Part II: Time-Temperature Dependent Matrix Behavior,' J. Compos. Mater. 27, 886-907 (1993a).

Miyase, A., S. S. Wang, A. W.-L. Chen, and P. H. Geil, "Anelastic Deformation of a Thermoplastic-Matrix Fiber Composite at Elevated Temperature; Part III: Structure and Thermomechanical Properties of AS4/ PACM-12 Composite,'” J. Compos. Mater. 27, 908-920 (1993b).

Mooney, M., "A Diffusion Theory of the Visco-Elasticity of Rubbery Polymers in Finite Elastic Strain," J. Polym. Sci. 34, 599-626 (1959).

Plazek, D. J., "Magnetic Bearing Torsional Creep Apparatus,'” J. Polym. Sci. A2 6, 621-638 (1968).

Pogany, G. A., “The $\alpha$ Relaxation in Epoxy Resins,” Eur. Polym. J. 6, 343-353 (1970).

Pomies, F. and L. A. Carlsson, "Analysis of Modulus and Strength of Dry and Wet Thermoset and Thermoplastic Composites Loaded in Transverse Tension,'” J. Compos. Mater. 28, $22-35$ (1994).

Rigby, Z., "The Value of Poisson's Ratio of Viscoelastic Materials," Appl. Polym. Symp. 5, 1-8 (1967).

Rouse, P. E., "A Theory of the Linear Viscoelastic Properties of Dilute Solutions of Coiling Polymers," J. Chem. Phys. 21, 1272-1280 (1953).

Schapery, R. A., "Stress Analysis of Viscoelastic Composite Materials," J. Compos. Mater. 1, 228-267 (1967).

Simon, P. P., "Modeling the Water-Modified Microstructure and Viscoelasticity of Epoxy Resins and Their Composites," Ph.D. dissertation, Texas A\&M University, College Station, Texas, 1995.

Slattery, J. C., Momentum, Energy, and Mass Transfer in Continua (Krieger, New York, 1981).

Tsenoglou, Christos, "Viscoelasticity of Rubber Networks with Dangling Chains," Polym. Prepr. Am. Chem. Soc. Div. Polym. Chem. 27, 290-291 (1986).

Tsotsis, T. K. and Y. Weitsman, "Energy Release Rates for Cracks Caused by Moisture Absorption in Graphite/Epoxy Composites," J. Compos. Mater. 24, 483-496 (1990).

Weiner, J. H. Statistical Mechanics of Elasticity (Wiley, New York, 1983). 